\title{
Forderungen an Politik, Wirtschaft und Gesell- schaft. Oder: Das Grundrecht des Kindes, nicht zu früh in die Kinderkrippe zu müssen
}

Serge K. D. Sulz, Alfred Walter, Florian Sedlacek

Wie bereits im Vorwort ausgedrückt, hat unsere Gesellschaft aktuell die unverzichtbare Aufgabe zu bewältigen, die Gleichberechtigung der Frau mit dem Mann voranzutreiben. Es fehlt noch sehr viel, bis eine gerechte Balance hergestellt ist. Alle Betroffenen müssen dafür Verzichte leisten: Der Mann muss seine Privilegien aufgeben und gleich viel Zeit und Verlangsamung seiner Karriere erbringen wie seine Frau. Das Unternehmen muss der Frau 18 oder 24 Monate Erziehungszeit geben und dazu dem Mann 12 oder 18 Monate bei Fortzahlung des Nettogehalts (das alternativ als Erziehungsgeld vom Staat gezahlt werden kann). Der Staat muss auf die von der Wirtschaft geforderte Maximierung der Zahl von Kinderkrippen verzichten und stattdessen teure qualifizierte und unschädliche Kinderkrippen finanzieren. Das Kind muss vielleicht schon mit 24 Monaten in die Kinderkrippe, während es gebraucht hätte, frühestens mit drei Jahren in den Kindergarten zu kommen. Die Träger von Kinderkrippen müssen darauf verzichten, große Gewinne auf diesem neuen Markt zu erwirtschaften. Wenn alle einen angemessenen und tragbaren Verzicht leisten, kann das Entweder-oder für die Frau (entweder zuhause am Herd oder meinen mir wichtigen Beruf) in ein Und verwandelt werden (zwei Jahre Kind und danach vierzig Jahre zur Berentung mein Beruf). Das sind $5 \%$ versus $95 \%$ der Zeit der Berufstätigkeit.

Es ist erstaunlich, dass die wissenschaftlichen Belege für den Nutzen und die Unschädlichkeit der Kinderkrippen von Forschungsinstituten kommen, die von der Wirtschaft finanziert werden.

Was für die Wirtschaft gut ist, für PolitikerInnen gut ist und auch für Frauen gut zu sein scheint, ist es für das Kind noch lange nicht. Wir wissen, dass wenn Wirtschaft und Politik etwas durchsetzen wollen, wissenschaftliche Erkenntnisse beiseitegeschoben werden. Knallharte Wirtschaftspolitik wird als menschenfreundliche Familienpolitik verkauft. Aus dem Familienministerium wird der verlängerte Arm des Wirtschaftsministeriums. Mit umfangreicher und cleverer Rhetorik wird den BürgerInnen via Dauerberieselung durch Presse, Funk und Fernsehen das Schlechteste als Bestes verkauft. Busse und Gathmann (2018) stellten allerdings fest, dass trotz starker Zunahme des Angebots und der Inanspruchnahme von Kindertagesstätten die Zahl in den Beruf zurückkehrender Mütter nicht zugenommen hat - die groß angelegte Aktion hat also die erwartete Wirkung nicht erreicht. 
Nach der Wende haben wir fast alles demontiert bzw. abgeschafft, was die DDR ausgemacht hatte. Nur eines nicht: die Kinderkrippen. Die hat die westliche Wirtschaft für sich entdeckt, um die Frauen so schnell wie möglich wieder an ihren Arbeitsplatz zu bringen. Und die PolitikerInnen waren wie immer dienstbar und hilfsbereit. Das schien gut zur Emanzipation der Frau zu passen, die auf ihren Beruf nicht mehr zugunsten der Mutterschaft verzichten wollte und sollte.

Da Kinder keine Lobby haben, die die Dinge anders sieht als ihre Eltern, sind sie die Leidtragenden. Das muss aber nicht so bleiben. Eltern können das Problem kurzfristig selbst in die Hand nehmen und langfristig für die notwendige Änderung der Politik sorgen - und sich vielleicht auch vom Diktat der Wirtschaft befreien.

\section{WAS KINDER BRAUCHEN UND VIELE ELTERN NICHT WISSEN: SICHERE BINDUNG}

Das Thema dieses Buchs steht und fällt mit der Erkenntnis, dass Kinder für eine gesunde geistige Entwicklung eine sichere Bindung zur Mutter und zum Vater brauchen. Das beginnt im Alter von acht Monaten mit dem Fremdeln. Zu diesem Zeitpunkt können Kinder unterscheiden zwischen Fremden und ihnen vertrauten Familienmitgliedern und sie können sich schon dagegen wehren, einem fremden Menschen in die Hand gegeben zu werden. Auch vorher schon, ab Geburt kann das Kind zwischen seiner Mutter und anderen Personen unterscheiden, aber wir merken es ihm oft nicht an. Der Aufbau einer sicheren Bindung beginnt also nicht erst mit acht Monaten, sondern läuft von Geburt an. Mit dem Laufen lernen mit einem Jahr kommt eine Schwierigkeit dazu: Denn das Kind bekommt einen Sinn dafür, dass es weglaufen und die Mutter dadurch verlieren kann oder dass die Mutter weggehen und es so die Mutter verlieren kann. Trennungsangst und Angst vor Alleinsein und Verlassenheit werden zur zentralen Angst. Bei Menschen mit einer unsicheren Bindung bleibt diese Angst ein Leben lang und bestimmt in großem Ausmaß die Beziehungs- und Lebensgestaltung, indem sie Autonomie, Selbständigkeit und Exploration der Welt bremsen und dadurch eine Chancenungleichheit bezüglich der Lebensqualität so früh schon bahnen. Daraus ergibt sich, dass das wichtigste Ergebnis der ersten zwei Lebensjahre eine sichere Bindung zu Mutter und Vater ist. Dazu gehören dass die Eltern bedingungsloses Willkommen sein, Geborgenheit, Schutz, Sicherheit und Zuverlässigkeit geben. Das ist eine lohnende Investition, denn dadurch wird das Kind ab zwei Jahren sehr neugierig auf seine Welt und beginnt diese zu explorieren und zu erobern. Seine wissbegierige Exploration (die nicht pädagogisch gefördert werden muss) führt dazu, dass es kognitive, kommunikative und emotionale Kompetenzen ganz von selbst entwickelt. Dazu ist keinerlei Erziehung nötig und keinerlei Bildungsmaßnahmen (die dürfen erst im Kindergarten beginnen). Es ist nur Beziehung nötig, die zu einer sicheren Bindung führt.

Leider vergessen alle Menschen, wie ihr Leben bis zum Alter von zwei Jahren war. Deshalb können sie sich auch nicht in die Psyche von Kindern in diesem Alter gut 
hineinversetzen. Und wir werden nie erfahren, wie genau es geschehen konnte, dass keine sichere Bindung entstand.

Aber es darf nicht schon wieder gegen die Mütter gehen, denn sie sind ebenso Opfer wie ihre Kinder: „In der neuen Krippendiskussion wird die kognitive Frühförderung überbetont und das emotionale Problem der Trennung von Mutter und Kind verleugnet. Insbesondere wird auch verleugnet, dass auch die Mutter beschädigt werden kann durch den frühen Trennungsdruck, dem sie nur ihre mütterliche Intuition, aber keine rationalen Argumente entgegensetzen kann." (Scheerer, 2014)

Es gibt zwar Eltern, die instinktiv wissen, was mit ihrem Kind los ist, was es braucht und was ihm schadet. Immer mehr Eltern haben dieses Feingefühl verloren. Das berichten Hebammen und Erzieherinnen. Einige können sich ihre Unsicherheit eingestehen und holen sich Hilfe und Rat. Andere gehen mit Vernunft und dem „gesunden" Menschenverstand verkopfter Erwachsener ran und sind fest überzeugt, dass sie es genau richtig machen und es ihrem Kind nicht schadet.

Ein großer Teil orientiert sich am Zeitgeist. Wenn so viele es so machen, dann muss das doch richtig sein. Und dann muss ich mich nicht durch scheinbar übertriebenes Mitgefühl mit dem Kind verunsichern lassen, sondern es so machen wie die Mehrheit, die das Sagen hat.

Dabei bleibt es aber nicht. Denn wenn sich Zweifel rühren und es vor den Freundinnen ausgesprochen wird, reagieren diese ablehnend bis heftig. Wer solche Freunde nicht verlieren will, wird dann nicht mehr darüber sprechen.

Nicht nur die Kleinstkinder sind sehr anpassungsfähig, sondern auch wir Erwachsene: In den fünfziger Jahren meisterten viele Eltern die informelle Norm einzuhalten, Kinder auf dem Balkon oder im Nachbarzimmer schreien zu lassen, bis es von selbst aufgehört hat, weil die Kinder sonst Tyrannen werden, denen man ewig ausgeliefert bleibt. Aber auch heute erbringen viele Eltern die Anpassungsleistung bravourös: Kinder kommen in die Kinderkrippe und Eltern müssen sich emotional ,abhärten', um nicht überfürsorglich zu sein.

Der große Bindungsforscher John Bowlby (1979) hat das Adaptionssyndrom beschrieben, bei dem Kinder, die anfangs schreien und weinen, sich auf einmal in ihr unabänderliches Schicksal fügen und still werden und brav und angepasst pflegeleicht. Jedes Kind hat ein angeborenes psychisches System, das eine sichere Bindung zu Mutter und Vater herstellt und so lange keine Ruhe gibt, bis das erreicht ist. Wenn Eltern sehr unzuverlässig sind, bleiben manche Kinder übermäßig anhänglich (was die Eltern nervt und die Tendenz hervorruft, das lästige Kind abzuschütteln, worauf dieses noch anhänglicher wird). Beim Adaptationssyndrom wird aber das Bindungssicherheitssystem überreizt und überfordert, so dass es im äußersten Fall zum Erliegen kommt. Das Kind versucht, ohne sichere Bindung zu überleben, es schafft es nicht einmal mehr zu klammern. Dazu fehlt ihm die Kraft. Wenn Eltern und ErzieherInnen dieses Ergebnis als gelungene Ablösung einschätzen, dann können beide beruhigt weitermachen mit einem Prozess, der Verletzung und Schädigung der kindlichen Psyche bedeutet. 
Die kognitive Entwicklung des Kindes im Vorschulalter konnte Fonagy (1997) aus der Bindungssicherheit mit der Mutter im Alter von 12 Monaten und mit dem Vater im Alter von 18 Monaten vorhersagen. $82 \%$ der sicher gebundenen Kinder lösten Theory-of-Mind-Aufgaben (reflektieren können, dass Überzeugungen und Wünsche eigenes Verhalten und das Verhalten anderer vorhersagen), während nur $46 \%$ der unsicher gebundenen Kinder diese Aufgaben lösen konnten. Ein anderes Studiendesign ergab, dass $87 \%$ der Kinder, die sowohl zu Vater als auch zu Mutter eine sichere Bindung hatten, diese Aufgaben lösen konnten, im Vergleich zu $63 \%$ der Kinder, die nur mit einer Elternperson eine sichere Bindung hatten, und nur $50 \%$ der Kinder, die zu keinem Elternteil eine sichere Bindung hatten. Fonagy schließt daraus, dass die kognitive Entwicklung bei sicher gebundenen Kindern früher die Fähigkeit einer Reflexionsfunktion im Sinne der Theory of Mind hervorbringt und damit der Entwicklungsprozess der Mentalisierung rascher vonstattengeht.

Um die Fähigkeit zu sicherem Bindungsverhalten zu erwerben, benötigt das Kind eine feinfühlige Mutter, die dem Baby seine Affekte spiegelt und es beruhigt (Fonagy et al., 2008). Damit die Affektspiegelung beruhigend wirken kann, muss sie sowohl den Affekt des Kindes treffend enthalten als auch die Information, dass die Mutter nicht so beunruhigt ist wie das Kind, sondern dass sie den Affekt gut meistern kann.

Wenn eine sichere Bindung hergestellt ist, muss das Kind keinen Aufwand mehr betreiben, um diese herzustellen, sondern wird frei für spielerische Entwicklung. Es kann und will sich früher kooperativen Interaktionsspielen zuwenden, wie sie die Als-ob-Spiele darstellen. Sie können Aufgaben zum Gedankenlesen und emotionalen Verstehen gut lösen (Astington \& Jenkins, 1995). Auch ältere Geschwister fördern die Mentalisierungsfähigkeit eines Kindes (Jenkins \& Astington, 1995). Die Entwicklung der Mentalisierung hängt auch von der eigenen Mentalisierungsfähigkeit der Mutter ab (Fonagy, Steele, Moran, Steele \& Higgitt, 1991).

Die ExpertInnen der Krippenforschung verkünden ständig, dass die Mutter-Kind-Bindung mit 12 Monaten abgeschlossen sei, weil man bei Studien feststellt, dass die Kinder in diesem Alter ein bestimmtes Bindungsmuster erworben haben. Dabei wird unterschlagen, dass sich die sichere Bindung im zweiten und dritten Lebensjahr festigen muss, um dem Kind das Gefühl von Sicherheit und Geborgenheit zu vermitteln. Dieser Prozess wird durch eine frühe Krippenbetreuung unterbrochen. Eine sichere Bindung zu den Eltern ist notwendig, um die Fähigkeit zur Impuls- und Affektregulierung zu entwickeln (Rass, 2017). Diese kann erst mit der Entstehung von inneren Bildern der Eltern im Alter von zwei Jahren stabil werden. Dazu gehören die unzähligen Forschungsarbeiten zum Thema Selbststeuerungsfähigkeit, über die Walter Mischel (2015) und Joachim Bauer berichten (2015). Denn die im Alter von vier Jahren vorhandene oder nicht vorhandene Selbststeuerungsfähigkeit (bei Kindergartenkindern am Beispiel des Belohnungsaufschubs untersucht $\rightarrow$ Marshmallow-Test) hat eine erstaunliche Vorhersagekraft auf Lebensqualität von 30- bis 35-jährigen Menschen:

„Vorschulkinder, die beim Marshmallow Test länger auf die Belohnung warteten, wurden Jahre später als Jugendliche folgendermaßen beurteilt: Sie zeigten mehr 
Selbstkontrolle in frustrierenden Situationen, sie waren nicht so anfällig für Verlockungen, sie ließen sich weniger leicht ablenken, wenn sie sich zu konzentrieren versuchten; sie waren intelligenter, selbstbewusster und zuversichtlicher, und sie vertrauten ihrem Urteilsvermögen. Unter Stress gerieten sie nicht so schnell in Panik wie diejenigen, die Belohnungen nicht zu lange aufschieben konnten, und sie verloren auch nicht so schnell die Fassung, waren nicht so leicht aus dem Konzept zu bringen und verfielen nicht so oft in unreife Verhaltensmuster. Ebenso konnten sie besser voraus denken und planen, und bei hinreichender Motivation waren sie zielstrebiger. Außerdem waren sie achtsamer, in höherem Maße zu rational-logischem Denken fähig und vernünftigen Argumenten aufgeschlossen, und ließen sich durch leichte Rückschläge nicht aus der Ruhe bringen.“ (Mischel, 2015, S. 38)

„Etwa im Alter zwischen 25 und 30 Jahren schafften es diejenigen, die im Vorschulalter länger auf die Belohnung warten konnten, laut eigener Auskunft besser, langfristige Ziele zu verfolgen, gefährliche Drogen zu vermeiden und ein höheres Bildungsniveau zu erreichen. Ihr Body Maß Index war zudem deutlich niedriger. Außerdem waren sie belastbarer und anpassungsfähiger bei der Bewältigung zwischenmenschlicher Probleme, und sie schafften es besser, enge Beziehungen aufrechtzuerhalten“ (a.a. O. S. 39).

„Bei den „guten Belohnungsaufschiebern“ war das Areal im präfrontalen Kortex aktiver, das für effektives Problemlösen, kreatives Denken und die Kontrolle impulsiven Verhaltens beansprucht wird. Dagegen war bei den Anführungszeichen „schlechten Belohnungsaufschiebern“ das ventrale Striatum aktiver, vor allem wenn sie sich bemühten, ihre Reaktionen auf emotional geladene, verlockende Stimuli zu kontrollieren. Dieses im tieferliegenden, primitiveren Teil des Gehirns angesiedelte Areal ist mit Verlangen, Lust und Sucht assoziiert. [...] dass „schlechte Aufschieber" offenbar einen starken Motor haben, während "gute Aufschieber" scheinbar eine bessere mentale Bremse besitzen“ (a. a. O. S. 41).

Im präfrontalen Kortex ist unser kühles System des logischen Denkens angesiedelt, es ist nicht schnell auslösbar, es reagiert relativ langsam - Überlegen braucht Zeit. Es reift erst allmählich und kommt erst ab 4 bis 6 Jahren zum immer effektiver werdenden Einsatz. Im limbischen System ist unser heißes System der Emotionen und Impulse zu Hause. Es ist schnell auslösbar, reagiert schnell reflexhaft, spontan, erzielt sofortige Wirkungen wie Befriedigung oder Spannungsreduktion. Es ist von Geburt an funktionsfähig. Große Bedürfnisse und großer Stress aktivieren dieses System.

Die zahlreichen von Mischel (2015) berichteten Studien aus dem Vorschulaltersbereich weisen eindeutig darauf hin, dass das menschliche Gehirn zum Zeitpunkt der Geburt noch nicht ausgereift ist und einige für unsere Betrachtungen wichtigen psychischen Prozesse zum Beispiel erst mit drei Jahren beginnen sich zu zeigen und erst mit fünf Jahren voll ausgebildet sind. Was ein Kind mit fünf Jahren kann, hat es zu einem guten Teil nicht erst gelernt, sondern es hat sich entwickelt. Die Individualität, die Persönlichkeit und die geistige Kapazität eines Kindes, wenn man es mit Gleichaltrigen vergleicht, ergibt sich zu einem großen Teil - neben seinen vererbten Merkmalen - aus den frühen Kindheitserfahrungen, die fördernd 
oder hemmend gewesen sein können. Wir müssen befürchten, dass Ganztags-Kinderkrippe vor dem Alter von 24 Monaten die Entwicklung des kindlichen Gehirns in oben genanntem Sinn hemmt.

Zepf und Seel (2017) berichten über zahlreiche Studien, die belegen, dass Kinderkrippen in den ersten zwei Lebensjahren Kindern schaden können. Auch die beste Kinderkrippe kann nicht verhindern, dass Kinder, die ganztags in einer Kinderkrippe untergebracht werden, einen sie überfordernden Dauerstress erleben. Dauerstress wiederum, so zeigen sehr viele Studien(wie die Zusammenfassung im Kapitel von Rainer Böhm und die in diesem Buch bereichteten Studien (Schulz \& Wulfe und Wedlich), hemmt die Entwicklung des Gehirns und damit auch die Entwicklung wichtiger geistiger, emotionaler und sozialer Fähigkeiten. Man muss deshalb damit rechnen, dass Eltern, die sich für eine Ganztags-Kinderkrippe vor dem Alter von 30 Monaten entscheiden, dazu beitragen, dass sich die Chancen ihres Kindes, sich seinen genetisch mitgegebenen Begabungen gemäß zu entwickeln und zu entfalten, deutlich verringern.

Wulfes und Schulz (2017) berichteten auf dem 35. Symposium der Fachgruppe Klinische Psychologie und Psychotherapie der Deutschen Gesellschaft für Psychologie über eine sorgfältig durchgeführte Studie über den Einfluss außerfamiliärer frühkindlicher Betreuung auf die Entwicklung von Verhaltensauffälligkeiten im Jugendalter. Es zeigten sich Verhaltensprobleme, externalisierende Verhaltensauffälligkeiten und geringeres prosoziales Verhalten. Sie empfehlen besonders frühes Eintrittsalter ( 0 bis 3 Jahre) zu vermeiden, außer bei Migrationshintergrund und belasteten Familien, wo die Integration über eine Institution besser gelingt als in der Familie. Sie betonen, dass die ersten Lebensjahre eine sehr wichtige Zeit im Leben eines Kindes sind.

Da wirkt es wie eine kleine Nebensache, wenn wie Butzmann (in diesem Buch) berichtet, sehr früh in der Krippe abgegebene Kinder zuerst zur Erzieherin Mama sagen statt zur Mutter. Oder was lästig wirken kann: Brisch \& Hellbrügge (2006) weisen darauf hin, dass Kinder, die keine Haupt-Bindungsperson finden (z. B. wegen häufig wechselndem Personal), „promiskuitiv“ sich Erwachsenen zuwenden, um sich körperliche Geborgenheit zu holen, was diese dann als distanzlos empfinden.

\section{WIR KÖNNEN DIE FORSCHUNGSERGEBNISSE SO ZUSAMMENFASSEN}

Die neurobiologische Basis der Entwicklung der Emotionsregulation und der Impulskontrolle ist die Reifung des Gehirns, speziell des präfrontalen Kortex u. a. mit seiner das limbische System modulierenden und hemmenden Wirkung.

Die Plastizität des Gehirns ermöglicht es der Umwelt, auf die Entwicklung in vielfältiger Weise einzuwirken - sowohl entwicklungshemmend als auch entwicklungsfördernd. Die zwischenmenschliche Umwelt ist die einbindende Kultur, die der Entwicklung des Individuums Raum gibt und Grenzen vermittelt. Entwicklung geschieht in Wechselwirkung mit ihr. 
Sichere Bindung zu den Eltern fördert die Reifung des Gehirns, unsichere hemmt sie. Abgeschlossen werden kann der Prozess des Aufbaus einer sicheren Bindung erst mit zwei bis drei Jahren, wenn Kinder in der Lage sind, ein inneres Bild der Eltern während deren Abwesenheit zu bewahren.

Kinder unter 24 Monaten brauchen nicht gleichaltrige sondern ältere Kinder und Erwachsene. Sie brauchen keine Gruppe, sondern Zweierbeziehungen (mit Mutter, Vater, anderen Erwachsenen, älteren Geschwistern).

Stress hemmt die Entwicklung des Gehirns. Gestresste Eltern stecken ihr Kind mit ihrem Stress an. Die Kinderkrippe ist toxischer Dauer-Stress für das Kind. Und die gestresste Eltern sind Stress für das Kind.

Wenn wir die Forschungsergebnisse der Bindungsforschung und der Säuglingsforschung studieren und ernst nehmen, wird klar, dass die mannigfaltigen Interaktionsprozesse zwischen Mutter und Kind und zunehmend auch zwischen Vater und Kind in Art und Ausmaß in den ersten zwei Lebensjahren ungestört ablaufen können müssen, ohne dass das Kind in Fremdbetreuung gegeben wird.

Hediaty Utari-Witt (2018) betonte in ihrem Vortrag wie wichtig es ist, die junge Mutter zu „bemuttern“ - zu unterstützen. Die Mutter kann etwas für sich tun, während Familie sich ums Kind kümmert. Oder - und das hebt sie ganz besonders hervor sie wird selbst bemuttert und versorgt.
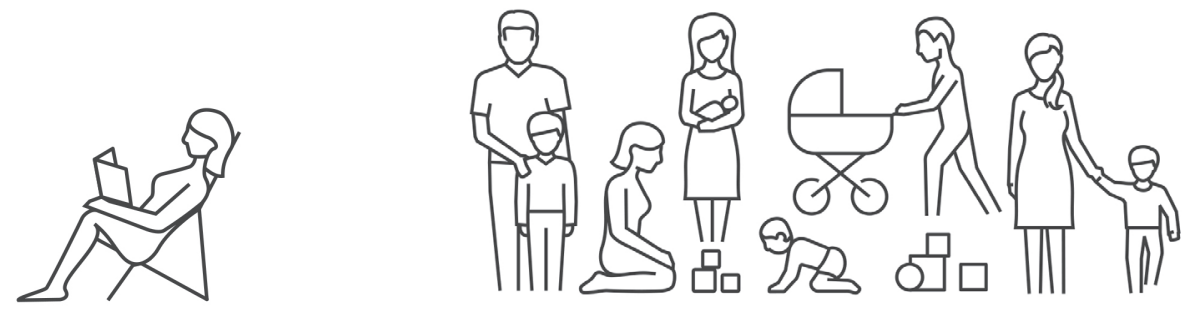

Wir vergessen sie oft - die Alleinerziehenden: Diana Schöniger leitet ihren Aufsatz über alleinerziehende Eltern mit einem afrikanischen Sprichwort ein: „Es braucht ein ganzes Dorf um ein Kind großzuziehen.“ Die Kinderkrippe ist das Gegenteil:
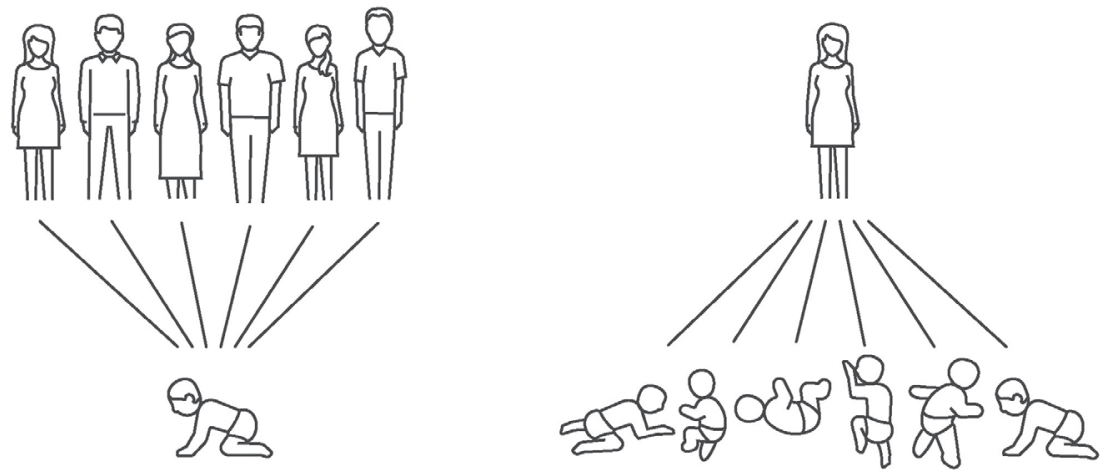
Sie weist darauf hin, dass viel für junge Eltern getan wird, aber wirklich nichts für Alleinerziehende, die sich nach dem Halt und der Unterstützung einer großen klassischen Familie sehnen, finanziell unterstützt zu werden, das Kind stundenweise abgeben zu können, weitere Bezugspersonen für das Kind zu haben etc. Sie fordert von der Wirtschaft flexible Arbeitszeiten und Arbeitszeitkonten und die Möglichkeit der Vernetzung auch in dem Sinne von Netz und doppeltem Boden für den ständigen Einzelkämpferkampf.

Junge Großstädterinnen insbesondere Akademikerinnen kennen fast nur Freundinnen, deren Kind in der Kinderkrippe ist. Vielleicht ist für sie die berufliche Karriere viel wichtiger. Ihr Frausein ist mehr mit ihrem Beruf verbunden als mit dem Muttersein. Sie wollen nicht so chancenlos und unzufrieden bleiben wie ihre eigene Mutter, die für die Kinder zuhause blieb, auf ihren Beruf ganz verzichtete und unglücklich war. Hebammen berichten, dass nicht wenige Eltern sehr unsicher und unbeholfen angesichts des Säuglings und Kleinkinds sind. Auch deshalb geben sie es gern in die zuverlässigen Hände einer professionellen Erzieherin. Nur leider ist es mit dem Versorgen nicht getan, wie Hans-Joachim Maaz in diesem Buch schreibt: „Entwicklungspsychologie, Bindungsforschung, Säuglingsforschung, Hirnforschung sind sich in einer zentralen Erkenntnis einig, dass die Qualität der frühen Beziehungsgestaltung mit dem Kind entscheidend für dessen Persönlichkeitsentwicklung ist. Das gestattet die gesicherte Aussage, dass es bei der Betreuung von Kindern nicht um Erziehung, sondern um Beziehung gehen muss. ... Zu beachten bleibt aber, dass die leibliche Mutter durch die frühesten Beziehungsverhältnisse in der Schwangerschaft, während der Geburt und in der Stillzeit eine bevorzugte Beziehungsbedeutung für das Kind hat, von der andere Betreuungspersonen (Vater, Großmutter, Tagesmutter, Krippenerzieherin) ausgeschlossen bleiben. Dieser besondere Beziehungsrang einer Mutter wird erst allmählich in der Entwicklung des Kindes relativiert und sollte nie zu früh oder zu abrupt aufgegeben oder infrage gestellt werden“.

Auf die Bedeutung der Mutterschaft im Leben einer Frau gehen Nadia Bruschweiler-Stern und Daniel Stern (2014) in ihrem Buch ein. Eine junge Frau ist zunächst noch weit vom Muttersein entfernt. Im Lauf der Schwangerschaft nähert sie sich diesem und mit der Geburt des Kindes ist sie auch als Mutter geboren.

\section{WIE KINDERKRIPPEN SIND UND WOVON DIE ELTERN NICHTS WISSEN}

Selbst Erzieherinnen sagen „Wenn die Eltern wüssten ...“ Dabei scheue ich davor zurück, Tatsachenberichte zu zitieren, weil der Leser die Missstände sogleich den Erzieherinnen anlastet, die aber nichts dafürkönnen und selbst darunter leiden. Es sei denn sie gehören zu denen, die den Rat eines Kinderkrippenleiters beherzigen: „Wenn Sie mit den Kindern mitleiden, haben Sie den falschen Beruf gewählt." Erika Butzmann schreibt in diesem Buch: „Eine berufserfahrene Krippenerzieherin berichtet, dass die Einjährigen in der Regel über ein halbes Jahr hinweg weitgehend auf dem Arm oder dem Schoß der Erzieherin verharren und erst dann sich langsam von ihr fortbewegen." So lange brauchen sie, bis sie das Mindeste an Bindung 
aufbauen konnten. Die uns vorliegenden Berichte der Erzieherinnen sprechen eine klare Sprache (Tichy in diesem Buch).

Wenn wir nicht von Erzieherinnen informiert würden, könnten wir nicht behaupten, dass trotz offiziellem Schlüssel von einer Erzieherin auf sechs Kinder viel zu oft eine Erzieherin zwölf bis sechzehn Kinder allein zu betreuen hat. Das kommt in nahezu allen Kinderkrippen vor. Krankheit, Urlaub oder Kündigungen führen dazu, ohne dass die Leitung das verhindern könnte. Die Mehrzahl der Kinder in der Kinderkrippe befindet sich trotz zeitweiliger Fröhlichkeit beim Spielen in einem toxischen Dauerstress, der die Entwicklung ihres Gehirns behindert. Wer will, kann diese Informationen vielfach nachlesen. Aber die Eltern, die ihr Kind in die Krippe getan haben, müssen sich vor dieser Wahrheit schützen. Wenn sie diese Tatsachen an sich herankommen lassen, schaffen sie es nicht mehr ihr Kind in die Krippe zu bringen, was aber die bisherige Gestaltung des Berufslebens radikal stoppen würde. Mann oder Frau müsste zuhause bleiben, ohne Erziehungsgeld. Die Miete könnte nicht mehr bezahlt werden oder der Kredit für die Eigentumswohnung. Das Auto müsste verkauft werden. Die Armutsgrenze wäre nicht mehr weit entfernt. Da aber erst $25 \%$ der Eltern im Westen (im Osten sind es 67\%) ihr Kind in die Kinderkrippe geben,

\section{DIE KINDERKRIPPE ERZEUGT TOXISCHEN DAUERSTRESS, DER DAS GEHIRN SCHÄDIGT}

Die für elterliche Entscheidungen wichtigste Wissensbasis ist die Stress-Forschung. Denn toxischer Dauerstress schädigt das kindliche Gehirn. Und viele Studien (siehe das Kapitel von Rainer Böhm in diesem Buch) konnten nachweisen, dass Kinder vom ersten Tag in der Kinderkrippe an unter einer erhöhten Cortisolausschüttung zu leiden haben, die toxisch auf Strukturen des Gehirns wie den Hippocampus wirken, der für die Gedächtnisorganisation zuständig ist. Diese Kinder werden später Entwicklungs- und Bildungschancen weniger nutzen können. Wohlgemerkt sind nicht alle Kinder betroffen, aber eine zu große Zahl.

Tierexperimentelle Studien ergaben, dass frühkindliche Traumatisierung (z.B. Mutterdeprivation) zu einer lebenslang erhöhte CRH-Produktion führt (CRH ist Cortisol-Releasing Hormon), das für die Produktion und Ausschüttung von Cortisol sorgt. Durch seine Erhöhung wird bei Stress mehr Cortisol ausgeschüttet (Nemeroff, 1996; Heim \& Nemeroff, 2001).

\section{Das Stresshormonsystem (HPA-Achse):}

Stress führt über

- Hippocampus,

- Amygdala,

- im Hypothalamus zu CRH-Ausschüttung, das

- in der Hypophyse (Vorderlappen) zur ACTH-(Corticotropin)-Ausschüttung führt (ACTH = Adrenocorticotropes Hormon), das

- in der Nebennierenrinde Cortisol (=Stresshormon) zur Ausschüttung bringt. 


\section{Cortisol bewirkt}

- muskuläre Energieversorgung (Blutzucker erhöht),

? Satz - psychische Alarmiertheit,

Irgend- • reduziert Hunger, Müdigkeit, Libido,

was

- verhindert akute Entzündungsprozesse,

komisch

- verhindert Temperaturerhöhung (Fieber),

oder Dop-

- reduziert Immunprozesse (Abwehr gegen Infektionen),

pelpunkt

- führt nach längerer Zeit zu Depression,

und Auf-

- wirkt bei Kleinkindern unter Dauerstress toxisch auf das Gehirn.

Trennung von der Mutter ist hochgradige Bedrohung und Frustration, die zu psychophysiologischem Stress führt. Die Psyche versucht auf drei Weisen Stress zu beenden (Abb. 1). Zunächst wird gekämpft durch Weinen und Schreien, auch durch Aggressivität. Wenn das nicht zur Stressreduktion führt, erfolgt Rückzug. Das ist die Variante der Flucht im Fight-Flight-Freeze-Mechanismus der Stressbewältigung, die dem Kleinkind möglich ist. Der Unterschied zum Freeze (völliges Erliegen jegliche auf die Außenwelt gerichteter Aktionen) ist gering. Die Kinder werden unscheinbar bis unsichtbar. Sie resignieren und erwarten keine Abhilfe von der Außenwelt mehr. Wenn auch dieser Mechanismus den Stress nicht wesentlich reduzieren kann, bleibt das Kind auf dem Stress und dessen Folgen sitzen: Krankheit und Depression. Allerdings gibt es viele Kinder, die so anpassungsfähig sind, dass sie durch maximale Anpassung an die widrigen Umstände weniger Stress empfinden müssen. Sie spüren kein Leid mehr und sind so pflegeleicht, dass Erzieherlnnen und Eltern zufrieden mit ihnen sind.

\section{Wege aus der Stressphase}

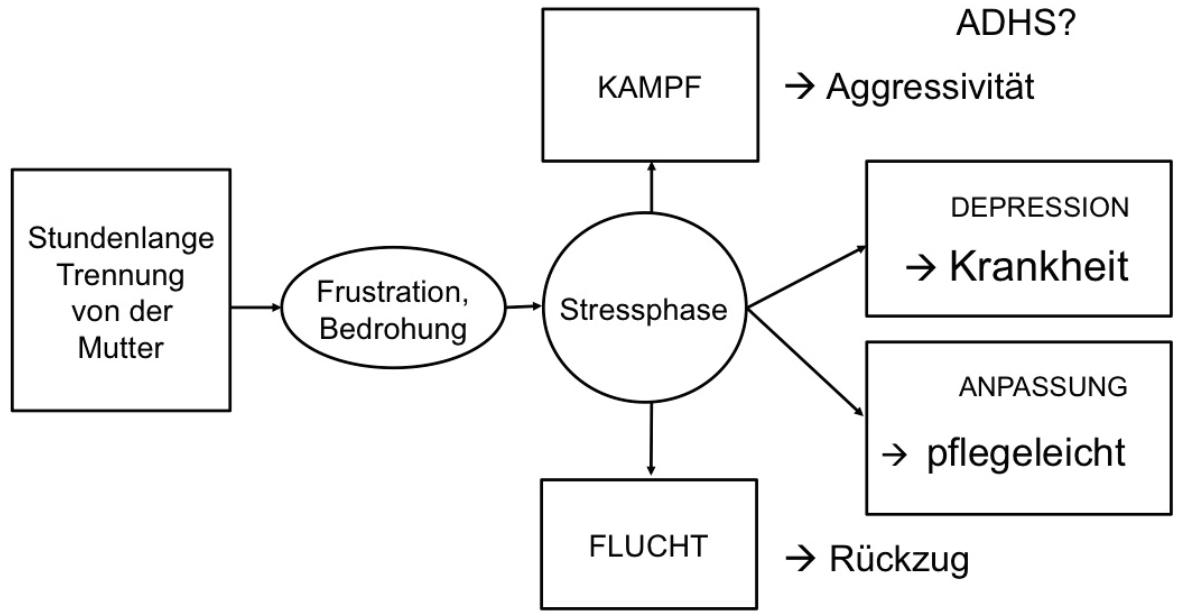

Abbildung 1 Wege aus der Stress-Phase (verändert nach Sulz 2017b,c) 
Wir müssen davon ausgehen, dass die Krippe auf wenige Kinder traumatisch und für sehr viele Dauerstress ist mit ständig erhöhter Cortisolausschüttung. Cortisol wirkt u.a. auf das Gehirn - auch neurotoxisch. Indirekt wirken Kinderkrippen deshalb wie ein Psychopharmakon (Sigman, 2011). Diese Wirkungen halten zumindest bis zum Alter von 15 Jahren an (längere Erfassungen gibt es noch nicht). Vandell et al. (2010) und Belsky \& Pluess (2011) stellten fest, dass aggressiv-dissoziales Verhalten umso häufiger ist, je länger die Kinder in der Kinderkrippe untergebracht sind. Noch mit 15 Jahren sind impulsiv-risikoreiche Verhaltensweisen häufiger.

Baker, Gruber und Milligan (2017) berichten, dass positive Effekte nur bei benachteiligten, gefährdeten Familien festzustellen sind, bei denen die Eltern keine ausreichende Betreuung der Kinder leisten konnten. In der repräsentativen Quebec-Studie ergaben sich keine positiven Effekte (weder kognitiv, noch sozial-emotional).

Belsky \& Pluess (2011) berichteten, dass das Kind umso länger in die Krippe gegeben wurde, je weniger feinfühlig die Mutter war.

Die neuesten Ergebnisse zu den Langzeitfolgen des Kinderkrippenaufenthalts brachte die sogenannte Quebec-Studie (Baker et al., 2017):

Krippenkinder wiesen später im Jugendalter mehr Kriminalität,

später im Jugendalter mehr Krankheiten,

später im Jugendalter weniger Lebenszufriedenheit auf.

Man beachte wieder die richtige Lesart: Die Aussage ist nur, dass der Prozentsatz schädlicher Langzeitfolgen statistisch signifikant erhöht ist und nicht, dass alle Kinder diese aufweisen.

Neben der kurzfristigen Erhöhung des Cortisolspiegels ist das Cortisol-Tagesprofil von großer Bedeutung. Morgens beim Aufwachen wird Psyche und Körper durch einen höheren Cortisolspiegel auf die Aktivitäten des Tages vorbereitet. Im Lauf des Tages sinkt der Spiegel physiologischerweise ab, so dass er abends so gering ist, dass das Einschlafen keine Probleme macht. Bei Kinderkrippenkindern wurde aber eine Umkehr des Tagesprofils beobachtet. Morgens wenig Cortisol (wenig Energie für den anstehenden Tag) und abends viel Cortisol (weil der Tag so stressig war). Der täglich anhaltende Dauerstress in der Krippe wird von Vermeer \& ljzendoorn (2006) mit externalisierenden Verhaltensauffälligkeiten in Verbindung gebracht und ist unabhängig von der Betreuungsqualität. Das Tagesprofil der Krippenkinder entspricht dem von rumänischen Waisenhauskindern (Gunnar, 2001, siehe Abb. 2).

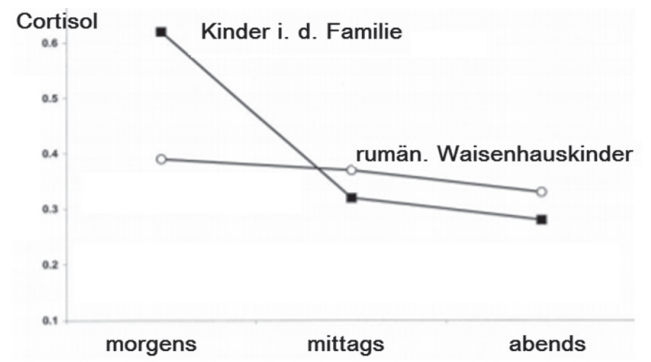

Abbildung 2 Umkehr des Cortisol-Tagesprofils bei rumänischen Waisenhauskindern (verändert nach Gunnar, 2001) 
Auch Roisman (2009) berichtet, dass Krippenkinder noch im Alter von 15 Jahre niedrige morgendliche Cortisolwerte haben, die den Werten familiär emotional vernachlässigter Kinder entsprechen. Eckstein et al. (2010) konnten die Umkehr des Cortisol-Tagesprofils vier Monate nach Beginn der Kinderkrippenbetreuung festhalten (Abb. 3).

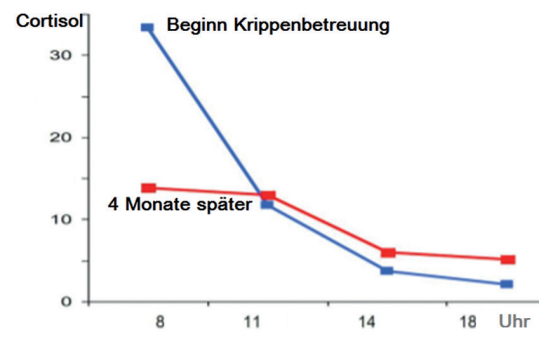

Abbildung 3: Umkehr des Cortisol-Tagesprofils nach 4 Monaten Kinderkrippenaufenthalt (Wiener Kinderkrippenstudie Eckstein et al., 2010)

Diese Profil-Umkehr erinnert an die scheinbar paradoxe Cortisol-Umkehr bei Trauma im Vergleich zur Depression, die ja einem Höchststand andauernder Cortisol-Ausschüttung entspricht. Die Neurobiologie kann diese Prozesse im Gehirn so nachvollziehen (siehe Sulz, 2017b,c):

Trauma:

$\mathrm{CRH}$ erhöht

Cortisol erniedrigt

Glucocorticoid-Rezeptorempfindlichkeit erhöht verringert
Depression

erhöht

erhöht

Inwiefern Trauma-Äquivalente bei Kinderkrippenkindern vorzufinden sind, müsste untersucht werden, z. B.:

als Ergebnis des völligen Sich-aufgebens,

des Erlebens von Unentrinnbarkeit und

der Chancenlosigkeit jeglichen Handelns

Stress wird zwar erlebt, aber Kampf- oder Fluchthandlungen werden nicht mehr durch Cortisol angestoßen.

Umgekehrt wissen wir, was kindlichen Stress reduziert (Sulz, 2017b,c):

- Gesicht der Mutter

- Stimme der Mutter

- Geruch der Mutter

- Körper der Mutter

- Brust und Säugen/Stillen der Mutter

- Wohlbefinden der Mutter (Stressfreiheit)

- Wiegen 
- Bedürfnisbefriedigung durch feinfühlige Mutter

- Beruhigung durch vertrautes Familienmitglied

- Befriedigt in Ruhe gelassen werden (allein sein dürfen)

Brisch (2009) betont, dass das Kind zunächst vor allem sensorische Stimulation und mütterlichen Körperkontakt braucht:

Zuerst braucht das Kind Stimulation über die Haut.

\section{Es braucht Körperkontakt.}

Dann über seine eigene Bewegung.

Und dazu über Sehen und Hören.

Auch später, wenn es nicht mehr um Bindungsaufbau, sondern um Exploration geht, braucht das Kind eine eins-zu-eins-Begleitung seiner Bindungsperson (was in der Krippe nicht möglich ist), bei der es sich durch Blickkontakt rückversichern kann (Brisch, 2009).

Nach der Bindung ist die Selbstwirksamkeit der zweite Eckpfeiler guter Entwicklung. Nach Brisch (2009) beginnen Kinder früh etwas selbst zu machen. Um ein Gelingen als Erfahrung von Selbstwirksamkeit zu speichern, brauchen sie jedoch das sehr erfreute Spiegeln der Mutter. In der heutigen Kinderkrippe hat die Erzieherin so viel zu tun, dass sie viel zu selten spiegeln kann. Die Motivation erlahmt, statt ein Gefühl von Selbstwirksamkeit entsteht ein Insuffizienzgefühl.

In den ersten zwei Lebensjahren wird also toxischer Dauerstress nur von der Mutter als primärer Bezugsperson wirksam beseitigt. Auch die besten Erzieherinnen können sie nicht ersetzen, zumal sie mit mehreren Kindern beschäftigt sind und laut Samel (in diesem Buch) sich nur 30 Minuten täglich dem einzelnen Kind ganz zuwenden können.

\section{QUINTESSENZ}

Weitere Berichte finden sich in den vorausgehenden Beiträgen. Wir können nun dazu übergehen, die Quintessenz zu konstatieren. Wir machen dies durch die Formulierung von Forderungen, die nicht wegen mangelnder Machbarkeit abgetan werden dürfen. Was unserer Gesellschaft wichtig ist, kann sie auch erreichen.

Wir werden hier einige Forderungen an die Politik, die Behörden, die Träger und die Wirtschaft formulieren, deren Realisierung zur Verbesserung der Kinderbetreuung beiträgt. Zuvorderst muss allerdings die Forderung stehen, dass das unselige Kinder- und Jugendhilfegesetz (Sozialgesetzbuch VIII) außer Kraft gesetzt wird:

§ 24 Anspruch auf Förderung in Tageseinrichtungen und in Kindertagespflege Ein Kind, das das erste Lebensjahr noch nicht vollendet hat, ist in einer Einrichtung oder in Kindertagespflege zu fördern 


\section{Die Forderungen im Einzeln, die nur für Kinderkrippen gelten, nicht für den Kindergarten:}

1a Forderung: Von einer Kinderkrippenbetreuung in den ersten zwei Lebensjahren sollte von Seiten der Regierung ausdrücklich abgeraten bzw. gewarnt werden.

1b Forderung: Drei Jahre Erziehungsgeld in der Höhe des bisherigen Nettogehalts (z. B. 1,5 Jahre für die Mutter, 15, Jahre für den Vater)

2a Forderung: Im dritten Lebensjahr maximal halbtags in eine qualifizierte Kinderkrippe. Generell sollte die Regierung von Ganztags-Kinderkrippen abraten.

2b Forderung: Im dritten Lebensjahr können die Eltern frei vereinbaren, wer wieviel beim Kind ist und wer wieviel arbeitet, z. B. arbeitet der Vater halbtags, die Mutter ganztags

3a Forderung: Eine Erzieherin ist für zwei Kinder präsent (Fachkraft-Kindrelation). Das entspricht einem Personalschlüssel von eins zu 1,5, da sie ja auch andere Aufgaben hat.

3b Forderung: Die ErzieherInnen dürfen möglichst nicht wechseln, so dass das Kind seine feste Bezugsperson behalten kann. Denn sonst macht es eine nicht zu bewältigende Trennungserfahrung.

4a Forderung: Alle ErzieherInnen müssen gut ausgebildet sein. D. h. es dürfen keine angelernten Hilfskräfte als Bezugsperson eingesetzt werden. Zudem muss jede Erzieherin eine U3-Zusatzausbildung nachweisen (Denn die Erzieherausbildung beinhaltet kaum dieBetreuung von Kindern von null bis drei Jahren).

4b ErzieherInnen müssen besser bezahlt werden, z.B. bei Fachhochschulabschluss wie GrundschullehrerInnen

5a Forderung: Sofortiger Zulassungs-Stopp für kindsschädliche Kinderkrippen, die derzeit das Angebot völlig dominieren (siehe Kinderkrippen-Ampel in diesem Buch)

5b Forderung: Schließung kindsschädlicher Kinderkrippen (siehe Kinderkrippen-Ampel in diesem Buch)

6a Forderung: Unsere Gesellschaft muss langfristig wieder zu einbindenden Kulturen für Eltern finden, die Kinderkrippen überflüssig machen

6b Forderung: Die Wirtschaft muss sich am Menschen orientieren. Sie ist für den Menschen da und nicht umgekehrt.

7a Forderung: Emanzipation der Frau muss neu definiert werden. Zur Gleichberechtigung mit dem Mann muss hinzukommen, dass sie für die Gleichberechtigung nicht ihr Muttersein verleugnen muss

7b Forderung: Wir müssen wieder zu menschen- und kinderfreundlichen Familienstrukturen finden. Das ist zwar nur ein Wunsch, aber wir sind überzeugt, dass die derzeitige Demontage der Familie gestoppt werden muss.

8a Forschung nur durch EntwicklungspsychologInnen, die sich auf die Entwicklung von null bis drei Jahre spezialisiert haben

$8 \mathrm{~b}$ Forschung nur unter Anwendung von direkter Beobachtung und Cortisolmessung in der Krippe 


\section{A FORDERUNG}

\section{Keine Kinderkrippe in den ersten zwei Lebensjahren}

Wir müssen nicht von einem familienpolitischen gestrigen Konservatismus ausgehen, der die Frau zurück an den Herd befördern will. Er reicht, die umfangreiche und vielfach bestätigte Forschung heranzuziehen. Wir müssen diese Forschung, die das ganze Kind im Blickfeld hat, unterscheiden von einer Scheuklappenforschung, die nur die intellektuellen Leistungen erfasst und unkritisch die massiv belastenden Anpassungsprozesse des überforderten Kindes schönredet. Es ist darauf zu achten, dass Forschungsaufträge und Forschungsgelder weniger die Interessen der Wirtschaft bedienen, wie das bei Studien der Fall ist, die nur die Anpassungsfähigkeit, die kognitive Entwicklung und den Ausbildungserfolg erfassen, also vorhersagen, wie gut ein Kind in Schule, Ausbildung und Beruf funktionieren wird, auch wenn das emotionale Leid der Kinder sichtbar und spürbar wäre.

Nicole Strüber und Gerhard Roth (2017) stellen die Frage: Lernt ein Kind die soziale Kompetenz nur durch die Krippe? Die Antwort der Wissenschaft ist: Das Kind wird nicht zum zurückgezogenen Sonderling, wenn es drei Jahre lang zu Hause betreut wird - vorausgesetzt es hat zu Hause eine feinfühlige Bindungsperson. Wir können noch etwas weitergehen: Es gibt keine dem Alter und dem Reifungsgrad des Kindes entsprechende Lernprozesse, die der Kinderkrippe bedürfen. Vor allem das Märchen, dass Kinder in diesem Alter gleichaltrige Kinder brauchen, ist durch Beobachtungsstudien widerlegt. Sie können noch nichts miteinander anfangen. Was da abläuft sind keine wertvollen Gruppenprozesse sondern Bewegen im Kollektiv, das Stress erzeugt. Nur auf mehrere Jahre ältere Kinder und auf Erwachsene sprechen die Kinder positiv an.

Wenn Kinder in den ersten zwei Lebensjahren nicht in die Kinderkrippe gegeben werden, bleibt nur eins: Mutter und Vater nehmen sich Erziehungszeit. Ideal wäre, wenn das in den ersten zwei Jahren die Mutter wäre, weil sich der Prozess des Schaffens von Bindungssicherheit anfangs auf die Mutter bezieht, wenngleich die Triangulierung der Beziehungen durch Einbeziehung des Vaters auch schon hier ein sehr bedeutsamer Prozess ist. Wir müssen der Bedeutung des Vaters einen sehr hohen Stellenwert beimessen. Auch die sichere Bindung zu ihm ist sehr wichtig. ER hilft dem Kind, sich aus der symbiotischen Dyade mit der Mutter heraus zu entwickeln zu einer Triade (Mutter-Kind-Vater), die die erste Brücke zu den späteren außerfamiliären Begegnungen und Beziehungen ist.

Damit die Mutter nicht 24 Stunden lang ihrem Kind "ausgeliefert" ist, ohne Chance ausreichender Befriedigung eigener Bedürfnisse, braucht sie Entlastung - durch den Vater, der trotz weiterer Ganztagstätigkeit sein berufliches Engagement reduziert und idealerweise tagsüber immer wieder durch weitere Familienangehörige, die so nah wohnen, dass sie schnell einspringen können - ein bis zwei Stunden am Tag. Oder die gegenseitige Aushilfe durch eine Freundin, eine Krabbelgruppe-Mutter oder eine Leih-Oma. Es unterliegt dem Elternpaar, für die Verfügbarkeit entlastender weiterer Bezugspersonen in diesem Sinne zu sorgen. So lange das nicht möglich ist, kann eine Halbtags-Kinderkrippe ab z. B. 24 bis 30 Monate helfen. 
D.h. ein Elternteil - die Mutter - bleibt die ersten zwei bis zweieinhalb Lebensjahre zuhause beim Kind. Das geht nur, wenn sie ausreichend Erziehungsgeld bekommt. Ausreichend ist dieses, wenn das Erziehungsgeld ihrem bisherigen Nettogehalt entspricht - bei voller Anrechnung auf die Rentenzeit.

Die Diskussionen in diesem Buch münden in zwei verschiedene Empfehlungen. Nachfolgendes Diagramm geht davon aus, dass ein Kind im Alter von 30 Monaten bereits halbtags in Fremdbetreuung gegeben werden kann (Abbildung 4). Es orientiert sich an der dgkjf-Kinderkrippen-Ampel (siehe obiges Kapitel).

Ein Thema, das noch nicht explizit angesprochen wurde: Die Aufklärungspflicht des Staates und der Träger - wie vor der Verabreichung von Medikamenten. Wir konnten feststellen, dass der Stress in der Kinderkrippe auf das Gehirn des Kindes neurotoxisch wirken kann. D. h., dass die Politik im Hinblick auf den weiteren Krippenausbau die Eltern aufklärt über die Risiken für die meisten Kinder. Damit erhalten die Eltern die Verantwortung zurück und müssen selbst entscheiden, ob sie das Risiko eingehen wollen. Damit erhalten die Kinder, für die eine Krippenbetreuung sinnvoll ist, weil deren Eltern nicht erziehungsfähig sind, eine hohe Qualität der Betreuung, weil weniger Kinder in den Krippen sind.

Kind in der Krippe: 30 - 12 Betreuung (dgkjf-Kinderkrippen-Ampel) dgkjf 2018
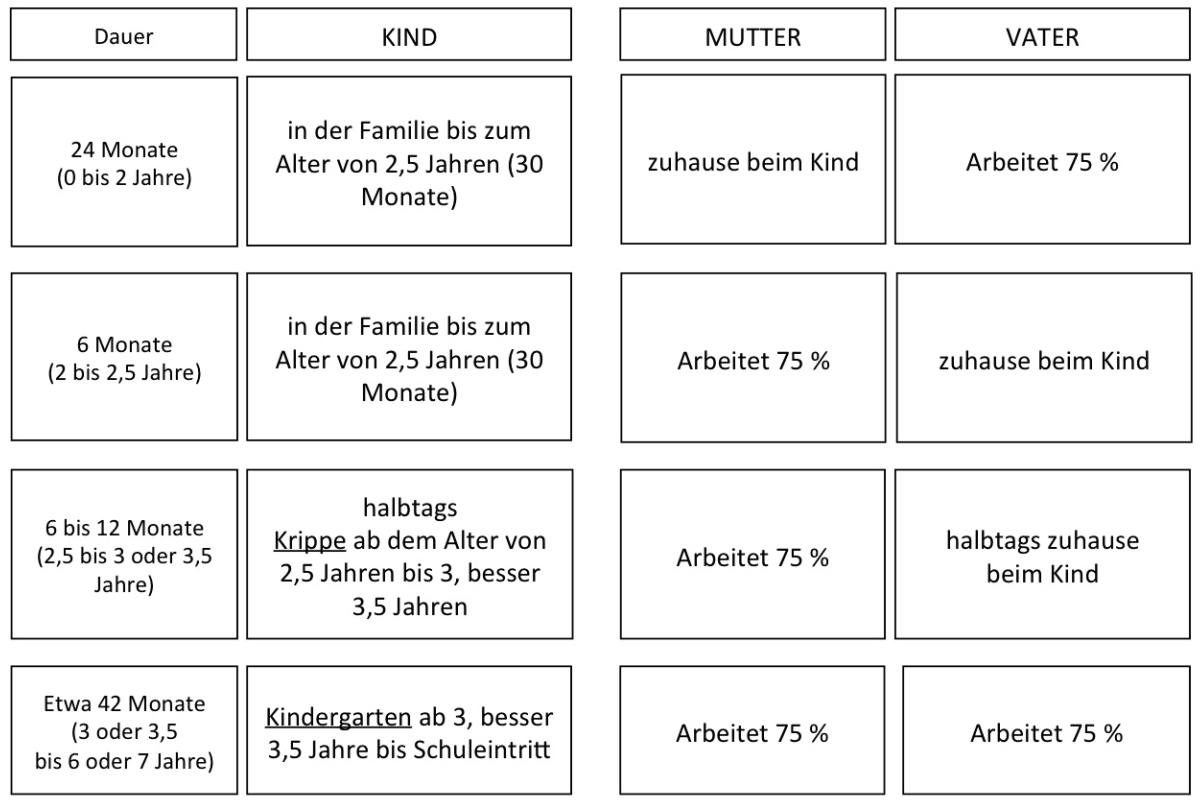

Arbeitet $75 \%$

Abbildung 4 Halbtags-Kinderkrippe erst ab dem 30 Monate. Mutter arbeitet dann wieder und Vater bleibt beim Kind (orientiert an der dgkjf-Kinderkrippen-Ampel) 
Dagegen stehen die Bielefelder Empfehlungen (Böhm, 2011, 2013a-c), die in Abb. 5 zum Ausdruck kommen.

Kind in der Familie: 18 - 18 Betreuung (Bielefeld) Böhm 2013

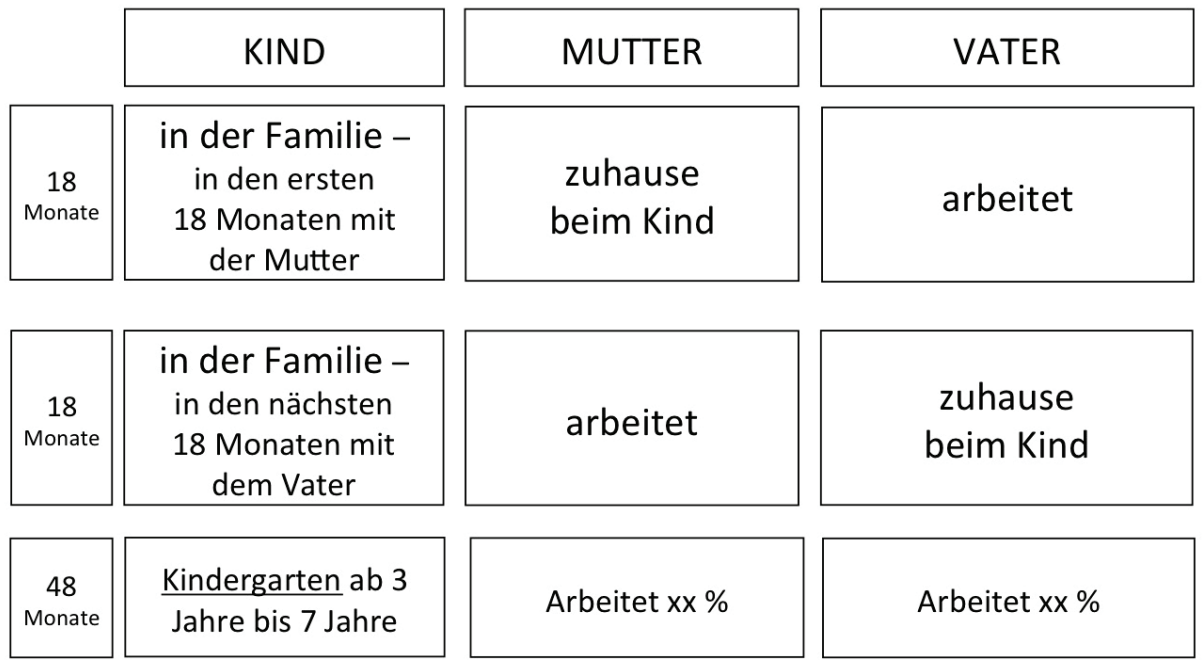

Abbildung 5 Das Kind bleibt bis zu seinem dritten Geburtstag in der Familie und geht dann gleich in den Kindergarten. Es besucht also keine Kinderkrippe.

\section{B FORDERUNG}

Zwei Jahre Erziehungsgeld in der Höhe des bisherigen Nettogehalts für die Mutter und ein halbes Jahr Erziehungsgeld für den Vater.

Zudem muss dafür gesorgt werden, dass keine Nachteile bezüglich der beruflichen Karriere entstehen. Arbeitgeber müssen gesetzlich verpflichtet werden, den Wiedereintritt in das Berufsleben ohne jegliches Festfrieren der Berufslaufbahn zu gestalten - mit Teilzeit, flexiblen Arbeitszeiten und Arbeitszeitkonten. Das sollte einhergehen mit der größeren elterlichen Verfügbarkeit auch im dritten Lebensjahr. Denn Kinder zwischen zwei und drei Jahren sind mit einer Ganztagskrippe immer noch überfordert. Auch sie erleben - wie viele Untersuchungen zeigen (siehe vorige Kapitel) -unzumutbaren Dauerstress, der ihnen leider oft nicht anzumerken ist. Ab dem Alter von 30 Monaten (2,5 Jahre) ist eine Halbtags-Kinderkrippe angemessen, wenn sie die genannten Qualitätskriterien erfüllt. Damit kommen wir zur

\section{A FORDERUNG}

\section{Im dritten Lebensjahr maximal halbtags in eine qualifizierte Kinderkrippe}

Da entsteht die Schwierigkeit, dass das Erziehungsgeld ausgelaufen ist und einer der beiden Elternteile in diesem Jahr auf halbtags reduzieren müsste. Wenn die 
Mutter die ersten zwei Jahre beim Kind bleiben durfte, ist jetzt der Vater dran, denn die Mutter muss sich jetzt wieder auf ihren Beruf konzentrieren können.

\section{B FORDERUNG}

\section{Im dritten Lebensjahr arbeitet der Vater halbtags, die Mutter ganztags}

Alles was notwendig ist, damit dieses Jahr familienverträglich ist, muss geregelt werden, an erster Stelle die Finanzen. Hier ist von Bedeutung, dass die Steuerlast der Familie drastisch reduziert wird, so dass beide zusammen das gleich Netto-Einkommen haben wie ein Doppelverdiener-Ehepaar ohne Kinder. Eine noch so subtile Bestrafung des Vaters bezüglich seiner beruflichen Chancen durch seinen Arbeitgeber muss gesetzlich unterbunden werden und zwar so, dass Arbeitsgerichte wirksame Schritte zur Herstellung dieses Aspekts von sozialer Gerechtigkeit einleiten können.

Dazu gehört aber auch eine Kehrtwende in der Einstellung des Vaters. Ein Hochschulprofessor wurde von der Kinderkrippenleiterin angerufen mit der Bitte, er möge sein Kind, das hohes Fieber bekommen hatte, abholen. Das Telefonat hat wirklich so stattgefunden:

Er: „Haben Sie meine Frau angerufen?“ Antwort: „Wir haben sie trotz mehrfachen Versuchen nicht erreicht. Können Sie das bitte machen?“ Er: „Was glauben Sie denn mit Ihrer Hausfrauen-Mentalität, was ich hier für eine Verantwortung habe? Ich bin mitten im Vorlesungsbetrieb und kann nicht weg. Was stellen Sie sich denn vor?" Vermutlich werden sich manche Männer beim Lesen nichts dabei denken, denn ein Mann hat Wichtigeres zu tun. Schon wird die Frau im Stich gelassen. Kinder sind Frauensache. Hier muss dem Mann abgerungen werden, dass Elternschaft eine faire Aufgabenteilung und eine faire Aufteilung des persönlichen Verzichts (auf Karriere, Einkommen, Sport, Hobby etc.) bedeutet. Fair ist: 50 \% übernimmt der Mann, egal wie viel mehr er verdient. Egal, wenn das seine Karriere etwas verzögert. Seine Karriere ist nicht wichtiger als Kind und Elternschaft.

\section{WIE KINDERKRIPPEN BEDÜRFNISGERECHT SEIN KÖNNEN}

Vor dem zweiten Geburtstag ist keine Kinderkrippe bedürfnisgerecht. Sie stört den Bindungsaufbau empfindlich, auch wenn sie gut ausgestattet ist. Die Eltern (besonders die Mutter) können in diesen beiden Lebensjahren nicht ersetzt werden. Eine Kinderkrippe, die Kinder unter 2 Jahren aufnimmt, ist demnach keine qualifizierte Kinderkrippe. Nach dem Aufbau einer sicheren Bindung zur primären Bezugsperson in den ersten zwei Lebensjahren kann das Kind beginnen, die Welt zu erkunden und zu erobern - durch Raum und Begrenzung gebende Betreuung einer Erzieherin als sekundäre Bezugsperson. Das dürfen aber noch nicht wechselnde Erzieherinnen sein. Das Kind braucht auch in diesem Jahr noch eine feste Bezugsperson, die ganz für sie und ein weiteres Kind da ist. 


\section{A FORDERUNG}

Eine Erzieherin ist für zwei Kinder präsent (Fachkraft-Kindrelation). Das entspricht einem Personalschlüssel von eins zu 1,5, da sie ja auch andere Aufgaben hat. Eine Kinderkrippe mit mehr als zwei Kindern pro Erzieherin (wirkliche Präsenz = Kind-Fachkraft-Relation), d. h. einem Personalschlüssel von mehr als eins zu 1,5 ist nicht qualifiziert.

Die Betreuerinnen dürfen nicht wechseln, sondern das Kind muss eine Bezugsperson haben, die ihm gehört und die es nur mit einem weiteren Kind teilen muss. Dass diese kurzzeitig immer wieder durch andere Erzieherin, die das Kind gut kennt, entlastet wird, gilt gleichermaßen wie die Mutter in den ersten zwei Lebensjahren regelmäßige Entlastung haben muss, indem Sie das Kind vorübergehend an eine dem Kind vertraute Bezugsperson aus dem nahen Familienkreis abgibt.

\section{B FORDERUNG}

Die Erzieherinnen dürfen nicht wechseln, so dass das Kind seine feste Bezugsperson behalten kann

Es muss verhindert werden, dass Bezugspersonen häufig wechseln wegen:

- Arbeitsbedingungen, die Erzieherinnen krankmachen

- Arbeitsbedingungen, die zu häufigen Kündigungen führen

- Schichtwechsel wie Früh- und Spätschicht

- Urlaub außerhalb der Ferien (stattdessen Betriebsferien während der Ferien)

Eine Kinderkrippe, die keine feste Bezugsperson in diesem Sinn gewährleistet, ist keine qualifizierte Kinderkrippe. Feste Bezugspersonen bedeutet, dass bei Krankheit der Erzieherin nicht eine andere Erzieherin einspringt, sondern dass sich die Mutter krankschreiben lässt. Das bedeutet auch, dass es wie bei Lehrern keine individuelle Urlaubsplanung außerhalb der Ferien geben kann, sondern dass die Kinderkrippe sechs Wochen lang pro Jahr (verteil auf verschiedene Ferien) Betriebsurlaub macht und dass in diesen sechs Wochen die Eltern ihr Kind ganz nehmen. Für Eltern gibt es in den ersten drei Lebensjahren keinen Urlaub ohne Kind.

\section{NUR WENN ES DER ERZIEHERIN GUT GEHT, KANN ES AUCH DEM KIND GUT GEHEN}

Eine qualifizierte Kinderkrippe zeichnet sich dadurch aus, dass eine Erzieherin Arbeitsbedingungen vorfindet, die eine erfüllende Ausübung ihres Berufs ermöglicht mit einer wertschätzenden und unterstützenden Leitung, einem sich gegenseitig unterstützenden Team, mit Zeit zum Austausch mit den Eltern, mit regelmäßiger Supervision und Fortbildung. Das Arbeitspensum muss ohne Überstunden gut zu bewältigen sein. Diese gesunderhaltenden Arbeitsbedingungen können durch externe Supervision am abgesichert werden.

Das pädagogische Anspruchsniveau ist umso höher je jünger das Kind ist. D. h. dass einer Kinderkrippen-Erzieherin mehr abverlangt wird als einer Kindergärtnerin und 
dieser mehr als einem Grundschullehrer. Dies muss sich in der Bezahlung abbilden, was bedeutet, dass eine Erzieherin so viel verdienen muss wie ein Grundschullehrer. Erzieherinnen kommt es zugute, wenn eine Erziehungsgemeinschaft mit den Eltern entsteht. Wie deren Partizipation möglich gemacht werden kann, diskutiert Textor (2014).

\section{A FORDERUNG}

\section{Alle Erzieherinnen müssen gut ausgebildet sein.}

Eine Kinderkrippe, die nicht alle Stellen mit voll ausgebildeten Erzieherinnen (Fachkräften) besetzt, ist keine qualifizierte Kinderkrippe.

D.h. dass ausgebildete Hilfskräfte (Ergänzungskräfte) in der Kinderkrippe nicht beschäftigt werden dürfen, auch keine angelernten Kinderpflegerinnen. Eine Kinderkrippe darf nur so viele Kinder aufnehmen, wie sie durch Fachkräfte betreuen kann!

Im Raum steht unsere Forderung, unverzüglich für alle Erzieherinnen, die mit Kindern unter 3 Jahren arbeiten, eine U3-Zusatzausbildung sofort einzurichten. Denn die Ausbildung als Erzieherin bereitet nicht auf diese sehr anspruchsvolle Tätigkeit vor, bei der kleine Fehler großen Schaden anrichten können.

Langfristig sollte der Beruf der Erzieherin auf Hochschulniveau angehoben werden (z. B. Hochschule für Angewandte Wissenschaften). Zu dieser Ausbildung gehört aber auch zwingend eine 100-stündige Gruppenselbsterfahrung. Ohne diese kann noch so anspruchsvolle theoretische Ausbildung nicht zu einem tiefen Verständnis des Kindes im ersten, zweiten und dritten Lebensjahr hinführen.

\section{B FORDERUNG}

Erzieherinnen müssen besser bezahlt werden, z. B. bei Fachhochschulabschluss wie ein Grundschullehrer

Bezahlung ist für eine erfüllte Berufstätigkeit nicht das wichtigste. Aber schlechte Bezahlung ist ein Zeichen geringer Wertschätzung. Wer schlecht bezahlt, bekommt kein gutes Personal.

\section{Fazit: Kinder müssen geschützt werden vor}

Krippen-Dauerstress, der die Entwicklung des Gehirns schädigt

Bindungsunsicherheit, die Chancenungleichheit für das Erwachsenenleben erzeugt

\section{A FORDERUNG}

\section{Sofortiger Zulassungs-Stopp für kindsschädliche Kinderkrippen}

Das kann nur gelingen, wenn sofort ein Zulassungs-Stopp für nicht in obigem Sinn qualifizierte Kinderkrippen erfolgt (das werden schätzungsweise nahezu $100 \%$ der gerade in Planung oder im Bau befindlichen Kinderkrippen sein). Thomas Rauschenbach, Matthias Schilling und Christiane Meiner-Teubner (2017) veröffentlichten aktuelle Berechnungen, die ein unverantwortliches Personal-Desaster erwarten lassen: Entweder Massenabfertigung der Kinder wegen fehlendem Personal oder angelernte Hilfskräfte, die die Entwicklungspsychologie der ersten drei Lebensjahre nicht verstehen können. 


\section{B FORDERUNG}

\section{Schließung kindsschädlicher Kinderkrippen}

Dazu gehört, dass unqualifizierte Kinderkrippen nach angemessener Übergangsfrist geschlossen werden, wenn es ihnen nicht gelingt, den notwendigen Personalschlüssel zu erreichen sowie obige Qualitätskriterien zu erfüllen. Richtschnur darf hier nicht das machbar Erscheinende sein, sondern das, was wir wirklich wollen. Da der Staat jeden Kinderkrippenplatz mit 1000 Euro monatlich subventioniert, kann dieses Geld ebenso gut denjenigen Eltern gegeben werden, die zweieinhalb Jahre lang bei ihrem Kind zuhause bleiben wollen.

\section{LANGFRISTIGE FORDERUNGEN: WERTSCHÄTZUNG UND SCHUTZ VON MUTTERSCHAFT UND FAMILIE}

Unsere Gesellschaft benötigt die Befreiung von der Versklavung durch die Wirtschaft, die Arbeitsbedingungen schafft, die immer menschenunwürdiger werden - ohne dass es die Betroffenen deutlich genug spüren. Viel zu viele Arbeitnehmer haben die Werte und Ziele der Wirtschaft übernommen und hinterfragen sie nicht mehr. Es ist doch selbstverständlich, für die nächste Beförderung in eine ferne Stadt umzuziehen, mitsamt der ganzen Familie und durch Verkauf des Eigenheims. Es ist doch selbstverständlich, dass zuerst die Arbeit kommt, dann ganz lang gar nichts und erst weit hinten die Familie. Die ins Extrem geschrumpfte Kleinfamilie ist für die Wirtschaft am besten zu handeln. Alles was soziales Leben im eigentlichen Sinn angeht und was ausschließlich in der Familie gelernt wird, bleibt auf der Strecke: das Miteinander, das Füreinander - auch über die Generationen hinweg. Individualität, Selbstwertsteigerung und Selbstverwirklichung geht nur noch über beruflichen Erfolg. Geldverdienen ist der Maßstab für diesen Erfolg. Genau solche Arbeitnehmer wünscht sich die Wirtschaft. Da es seit langem auch die Frauen erfasst hat, kommen jetzt die Kinder immer öfter unter die Räder. Und in der Tat, je ähnlicher wir in dieser Hinsicht den erfolgreichen asiatischen Ländern (Japan und China) werden, umso eher ist der Erfolg unserer Wirtschaft gesichert - auf Kosten der Menschlichkeit.

Wie sehr wir dennoch oder vielleicht sogar noch mehr Bestandteil eines Kollektivs sind, mit dem wir schwimmen, ohne wirklich individuell und bewusst selbst zu entscheiden und unser Schicksal wirklich in die eigene Hand zu nehmen, müssen wir immer wieder erschreckt feststellen, wenn - ohne dass man gemerkt hat, wie es kam - Trends entstehen und unglaublich viele diesen Trends folgen. Seien es Moden, politische Strömungen oder Lebensstil-Tendenzen. Also ist Individualismus nicht mehr als eine Ausgeburt des Kollektivs und damit nur scheinbare Individualität.

Wir Europäer sind zu denaturierten Menschen geworden, die ihre Natur nicht durch zivilisierte Höherentwicklung hinter sich gelassen haben, sondern in deren Verballhornung gelandet sind. Ob nun der Kaiserschnitt für die heutige Geburt zur Norm wird, oder wir überhaupt unseren Körper nur noch dazu verwenden, unseren Kopf zu tragen, wir haben unsere Leibhaftigkeit verloren. So wie wir unsere natürlichen Lebensgemein- 
schaften verloren haben. Das Zusammenleben mit der Familie mit Großeltern, Eltern, Onkel und Tanten als einbindender Kultur im Sinne von Robert Kegan (1986). Wir sind so denaturiert wie haltbare Milch im Tetraeder, von der man sagt, sie sei gar keine Milch mehr, die einzige Gemeinsamkeit mit Milch sei die weiße Farbe.

Ein Kind braucht aber eine Mutter in einer einbindenden Kultur (Kegan 1986), in der sie unterstützt wird, zu der sie gehört und nicht mit dem Kind alleingelassen wird. Nur dann kann diese Mutter für das Kind die alters- und entwicklungsgerechte einbindende Kultur werden. Wir brauchen gesellschaftliche Veränderungen, die wieder einbindende Kulturen schaffen, ohne künstliche Strukturen, die vom Staat und Institutionen geschaffen werden. Die Wirtschaft braucht den denaturierten Menschen und der Staat sorgt für diese Denaturierung, wie das Beispiel der Kinderkrippen für Kinder unter 2 Jahren zeigt.

\section{A FORDERUNG}

Unsere Gesellschaft muss wieder zu einbindenden Kulturen für Eltern finden, die Kinderkrippen überflüssig machen

Dazu ist eine Erneuerung der Wertorientierung der Menschen in unserer Gesellschaft nötig. Damit sie sich wieder wünschen, zwei bis drei Jahre in der Familie beim Kind zu bleiben, statt sich einen Rechtsanspruch auf einen Krippenplatz ab 1 Jahr zu erkämpfen, damit sie schnell wieder Doppelverdiener werden können.

Das kann nur gelingen, wenn der Wirtschaft Einhalt geboten wird und sie sich nicht mehr des Staates bedienen kann, um ihre Interessen den Bürgern aufzuoktroyieren, z. B. durch massenhaften Bau von Kinderkrippen.

\section{B FORDERUNG}

Die Wirtschaft muss sich am Menschen orientieren. Sie ist für den Menschen da und nicht umgekehrt.

Es darf nicht sein, dass Karriere den Umzug der ganzen Familie in eine 500 km entfernte Stadt notwendig macht. Firmen müssen ihrem Mitarbeiter in der Region eine Möglichkeit schaffen, sich weiter zu entwickeln. Es darf auch nicht sein, dass einige Familien keine andere Option haben, als ihre Kinder früh in Krippen betreuen zu lassen, weil sie von einem Gehalt nicht mehr leben können oder alleinerziehend sind. Die Politik hat die Aufgabe, das zu verändern: Aus der Herrscherin Wirtschaft die Dienerin Wirtschaft zu machen.

\section{A FORDERUNG}

Emanzipation der Frau muss neu definiert werden. Zur Gleichberechtigung mit dem Mann muss hinzukommen, dass sie für die Gleichberechtigung nicht ihr Muttersein verleugnen muss

Es fehlt noch viel bis zum Erreichen wirklicher Emanzipation der Frau in unserer Gesellschaft. Frauen müssen weiter darum kämpfen. Einerseits gibt es Zeichen dafür, dass ihr Kampf derzeit ein Siegeszug werden kann: Mädchen sind besser in der Schule, Mädchen sind sozial kompetenter. Frauen haben beginnen häufiger ein Studium. Frauen sind bei mehreren Numerus-clausus-Studienfächern in der Über- 
zahl. Und sie übernehmen auch die Mehrheit in immer mehr Berufen. Genau an dieser Stelle hört es dann aber auf: Wenn es um Stellenbesetzungen und um Beförderungen, um Spitzenpositionen geht, sitzen die Männer noch fest im Sattel. Ja und dann kommt die große Chancenungleichheit: Die Vereinbarkeit von Familie und Beruf geht wohl nur Frauen etwas an. Frauen tragen die Verantwortung für die Elternschaft - wie eine Last, durch die sie im Wettrennen um berufliche Karriere keine Chance gegen die männlichen Konkurrenten haben. Da diese ihnen die Last nicht abnehmen, müssen sie sich dieser Last auf andere Weise entledigen: Durch die Kinderkrippe. Diese befreit Frauen von einer Bürde, die ihnen althergebrachte Traditionen auferlegt haben. Sie bringt endlich die Befreiung, um ihr eigenes Leben genauso frei gestalten zu können wie ein Mann. Naja, sagen wir mal "fast so frei“. Selbstverwirklichung war früher eine Sehnsucht, ein Traum. Heute ist sie zum Greifen nah. Und unsere Gesellschaft fördert sie und sichert das Recht darauf gesetzlich ab. Schon in früheren Jahrhunderten haben adlige, gebildete bzw. reiche Frauen die Versorgung ihrer Kinder abgegeben, angefangen von der Amme, die das Stillen übernommen hat, über das Kindermädchen bis zur Gouvernante. Für diese Frauen waren nicht die Kinder selbst eine Last - nein, sie waren Mutters Freude. Allein die zeitaufwändige Versorgung der Kinder war lästig und entsprach nicht ihrem Anspruch und Status. Heute geben Akademikerinnen ihre Kinder am häufigsten und frühesten in die Kinderkrippe. Nicht Frauen an sich, nicht alle Mütter, aber die besonders bildungs- und leistungsorientierten Akademikerinnen sind in der gleichen Gefahr wie diese privilegierten Frauen früherer Jahrhunderte. Sie laufen Gefahr, einen wichtigen Teil ihres Frauseins zu verlieren: das Muttersein. Sie gehen so sehr in den Intellekt, dass sie den Bezug zu diesem Teil ihrer Körperlichkeit verlieren. Stattdessen wird der Körper in die gegenteilige Richtung (wiederum leistungsorientiert) durch Sport verschiedenster Art ertüchtigt. Körperliche Mutterschaft hört nicht nach der Geburt des Kindes auf. Das Stillen, das Wiegen, das Tragen, das Schmusen, das Liebkosen, das für das Kind Greifbar-sein, das Schützen, das Begrenzen. Ebenso der Geruch der Mutter, ihre Stimme, ihre Haut, ihre Bewegungen, ihre Handlungen. Das alles wäre nicht nötig, wenn das Menschenkind nicht im Sinne der vergleichenden Verhaltensforschung eine „Frühgeburt“ wäre und so unfertig auf die Welt käme, dass nicht wenige Wochen wie bei anderen Säugern reichen, bis das Kind vergleichsweise wenig umsorgt werden muss. Menschenkinder brauchen stattdessen zwei bis drei Jahre weitere Mutterschaft, die leider in einer Kinderkrippe nicht möglich ist. Aber was wäre der Mensch, wenn er sich über die Jahrtausende hinweg nicht immer wieder aufgebäumt hätte gegen die Grenzen, die ihm seine biologische Herkunft und Natur gesetzt hat. Ob es die Überwindung der Ozeane ist oder der Flug ins Weltall. Wir akzeptieren keine Grenzen. Wir überwinden die Natur. Der Fortschritt kann nicht aufgehalten werden. Unsere Wirtschaft bedient sich des Fortschritts und - leider auch - der Emanzipation der Frau. Diese wird nach der Geburt so schnell wie möglich wieder als Arbeitskraft verfügbar. So können wir fragen, ob die praktische Idee mit den Kinderkrippen der Emanzipation der Frau entsprungen ist oder ob die scheinbare Emanzipation der Frau mit dem Instrument der Kinderkrippe von den Unternehmen dazu benutzt wird, um sich 
der Arbeitskraft der Frau maximal bedienen zu können. Sie wird ausgebeutet und meint, es sei Emanzipation. Sie gibt ihr Mutter-sein als einem wichtigen Teil ihres Frauseins preis und meint, es sei Emanzipation. Ein sehr hoher Preis.

\section{B FORDERUNG}

\section{Wir müssen wieder zu menschen- und kinderfreundlichen Familienstrukturen finden} Wenn Zivilisation Denaturierung bedeutet, also Ablegen des biologischen Teils unserer menschlichen Existenz, dann ist das Dekadenz und diese bedeutet zwangsläufig einen Niedergang. Die Entwicklungsgeschichte zeigt deutlich in welche Richtung der Homo sapiens sich entwickelt hat. Der Kopf ist im Vergleich zu anderen Säugern größer. Er ist so groß geworden, dass er bei der Geburt fast nicht mehr durch den Geburtskanal passt. Die immer häufiger werdenden Kaiserschnitt-Geburten weisen darauf hin - wobei diese Zunahme ein Resultat der Risikominimierung von Seiten der Geburtshelfer bedeutet.

Unsere Gesellschaft wird beherrscht von der Wirtschaft. Der Mensch ist dazu da, ihr zu dienen. Politiker sind nur Mediatoren, deren Vermittlung aber im Zweifelsfall Entscheidungen stets zugunsten der Wirtschaft entstehen lassen. Das wird nicht nur bei der Automobil- und Tabakindustrie deutlich, sondern eben auch bei der Anpassung der Familienstrukturen an die Bedürfnisse der Wirtschaft. Da wird das Eigenheim verkauft, familiäre und freundschaftliche Bindungen gelöst, den Kindern ihre Freunde genommen, ihre Schulkameraden, ihre Schule - nur um den nächsten Karriereschritt zu gehen. Auch die Ehefrau muss ihre Arbeitsstelle aufgeben, ohne zu wissen, ob sie am neuen Wohnort eine Arbeit finden wird. Umgekehrt passiert das selten: die Karriere der Frau ist nicht so häufig Anlass für diese unnatürliche Mobilität. Ja Mobilität und Flexibilität ist gefragt, wenn man heute erfolgreich sein will. Und je kleiner die Familie, umso leichter geht der Umzug vonstatten. Die heutige Kleinfamilie ist die ideale Familienstruktur, um die Wirtschaft bestens bedienen zu können. Dann nimmt man die Kinderkrippe dazu und schon haben wir eine perfekte Dienstleistung für die Wirtschaft kreiert.

Das kann man aber nicht mehr Familie nennen. Das ist kein Familienleben. Aus Familiensicht ist das tot, so tot wie ein Schrumpfkopf im Vergleich zu einem lebenden Kopf. Aber solange das Individuum mit seinem Drang nach Selbstverwirklichung die gleichen Interessen hat, wie die Wirtschaft, wird das so bleiben. Die Selbstverwirklicher bekommen, was sie brauchen und die Wirtschaft bekommt, was sie braucht. Nur Familienmenschen bleiben auf der Strecke - und die Kinder.

Wir opfern menschliche Werte, die nur durch Familie und soziale Gemeinschaft verwirklicht werden können. Wir müssen wieder zu wirklichen Familien finden. Diese können durch Blutsverwandtschaften gekennzeichnet sein. Heute ist es aber genauso naheliegend, Wahlverwandtschaften einzurichten. Menschen, die sich mögen und die die gleichen Vorstellungen vom Leben haben. Statt den drei oder vier Menschen der Kleinfamilie leben z.B. acht oder zwölf Menschen zusammen. Und um sie herum ist eine quasi dörfliche Nachbarschaft, die sich zu ihnen gehörig fühlt und die die Kinder als zugehörig kennen und empfinden.

Wir müssen für unser qualitativ menschliches Überleben zum Klimawandel den Fa- 
milienwandel hinzufügen. Dieser bedeutet aber, dass nicht mehr der Mensch für die Wirtschaft da ist, sondern die Wirtschaft für den Menschen. Deshalb wird der Hauptwiderstand wie beim Klimawandel von der Wirtschaft kommen - nicht offen, sondern mit Hilfe der Politiker.

\section{A FORDERUNG}

Forschung nur durch EntwicklungspsychologInnen, die sich auf die Entwicklung von null bis drei Jahre spezialisiert haben

Die bisherige Forschung wurde von den falschen Forschern durchgeführt: Forscher, die keine EntwicklungspsychologInnen sind, die sich auf das Alter von null bis drei Jahren spezialisiert haben, sondern Wirtschafts, Sozial- oder epidemiologische Forschung betrieben und ganz neu in diesem Bereich sind. Oder Pädagogen, die den Bildungsaspekt oder Erziehungsaspekt auf U3-Kinder anwenden wollen. Es geht aber in diesem Alter nicht um Bildung und auch nicht um Erziehung, sondern um Beziehung und kenntnisreiche Interaktion, die das fördert, was beim Kind angelegt ist, statt Trainings an es heranzutragen. Vor allem muss die Forschung von Instituten durchgeführt werden, die nicht von der Wirtschaft finanziert sind.

Es fällt auf, dass nicht wenige Studien zum Nutzen von Kindertagesstätten von Wirtschaftsinstituten oder von Instituten kommen, die von der Wirtschaft finanziert werden. Welchen Nutzen hat also die Wirtschaft davon? Offensichtlich sind ihr qualifizierte Arbeitskräfte schnell wieder verfügbar. Es gibt exakte Rechnungen, die belegen, wie viel größer der Gewinn der Wirtschaftsunternehmen ist, wenn Kinder schon im ersten Lebensjahr in die Krippe gegeben werden und die Mutter wieder ganztags arbeitet. Um diese Gewinnchancen zu sichern, muss den Eltern und der Politik belegt werden, dass vor allem die Kinder davon profitieren. Da ist das Geld, das in wissenschaftliche Studien gesteckt wird, eine lohnende Investition. Und es wurde auch ausgerechnet, um wieviel sich die Steuereinnahmen des Staates erhöhen, wenn Kinder so früh wie möglich in die Krippe gesteckt werden - eine der lohnendsten staatlichen Investitionen - übrigens auch für Kommunen und Träger. Ein boomender Geschäftsbereich.

Die Leiterin einer Studie des Deutsches Institut für Wirtschaftsforschung, fasst das Ergebnis ihrer so zusammen: „Es wäre jetzt, auch vor dem Hintergrund unserer Ergebnisse, sicherlich wünschenswert, das Angebot der Nachfrage für die Ein- bis Dreijährigen anzugleichen und damit die Plätze zur Verfügung zu stellen, die die Eltern nachfragen, weil positive längerfristige Effekte eines frühen Kita-Besuchs auch für unter Dreijährige naheliegen“ (www.diw.de/interview). Diese Aussage ist methodisch unqualifiziert, da sie keinerlei Aussagen über eine Altersgruppe machen kann, die sie nicht untersucht hat.

Man muss zudem unterscheiden:

a) wie groß das jetzige Leiden der Kinder in der Krippe ist (toxischer Dauerstress bei unter Dreijährigen)

b) zu welchen späteren Kompetenzen der Krippenaufenthalt führt (Extraversion wobei nicht alle extravertierten Menschen die so sehr gelobte hohe Durchsetzungsfähigkeit haben). 
So könnte es rein hypothetisch sein, dass eine Operation ohne Narkose unerträglichen Schmerz und massive Traumatisierung bedeutet, dass aber später sehr resistente Menschen aus den betroffenen Kindern werden, z.B. gute Einzelkämpfer, sehr gute Manager, sehr gute Sportler (denn ein Indianer kennt keinen Schmerz). Wenn wir sagen, der Zweck heiligt die Mittel, dann sorgen wir dafür, dass bei so vielen Menschen solche grausamen Operationen durchgeführt werden, wie wir Profi-Sportler, Elite-Soldaten, TOP-Manager und Pioniere der Wissenschaft brauchen. Das ist dann das Beste für unsere Gesellschaft.

Wenn diese Operationen zwischen der Geburt und dem Alter von zwei Jahren durchgeführt werden, können wir garantieren, dass der Betreffende sich später nicht mehr daran erinnern wird. Die neurobiologische Wissenschaft kann begründen, weshalb das so ist.

Fazit: Studien zur eventuellen Schädlichkeit von Kinderkrippen dürfen nicht von wirtschaftlichen Interessen geleitet sein. Sie müssen von denjenigen entwicklungspsychologischen Forschern durchgeführt werden, die auf die Entwicklung zwischen null und drei Jahren spezialisiert sind. Und sie dürfen auch nicht von pädagogischen Forschern durchgeführt werden, die die Begriffe Erziehung und Bildung in den ersten drei Lebensjahren unterbringen wollen. Denn diese beiden Zielrichtungen disqualifizieren sie bezüglich der Grundkenntnisse der U3-Entwicklungspsychologie. In den ersten drei Lebensjahren geht es nicht um Erziehung, sondern um Beziehung. Und es geht nicht um Bildung, denn das kindliche Gehirn ist noch gar nicht reif und empfangsbereit für solche Maßnahmen.

\section{B FORDERUNG}

Forschung nur unter Anwendung von direkter Beobachtung und Cortisolmessung in der Krippe

Fragebögen, die Eltern ausfüllen sollen, führen zu unreliablen und invaliden Ergebnissen und sollten wegen der irreführenden Information der Allgemeinheit unterlassen werden. Denn Eltern dürfen kein Mitgefühl mit ihrem Kind haben, sonst müssten sie es aus der Krippe rausnehmen. Nur direkte Beobachtung des Kindes und Cortisol-Speichelproben ergeben zuverlässige und gültige Forschungsdaten.

\section{SCHLUSSWORT}

Während die Politik Gas gibt und zigtausend neue Kinderkrippen entstehen, mit viel zu wenig ausgebildetem Personal und viel zu viel unqualifiziertem Personal, führt sie einen wahren Infarkt der Kinderversorgung herbei. Das Gesetz von 2013 hat auf den Kopf gestellt, was als notwendige Kinderversorgung gilt. Mit dem Recht der Eltern auf einen Kinderkrippenplatz wird ihren Kindern Unrecht getan.

Statt einen Kinderkrippenplatz einzuklagen, sollten Eltern verlangen, dass sie die Möglichkeit bekommen, die ersten zweieinhalb Lebensjahre bei ihrem Kind bleiben zu können. Das sollten sie einklagen, nicht das Gegenteil. Falls Kinder ein 
Grundrecht haben, dann verstößt dieses Gesetz gegen ihr Grundrecht - auf einen Lebensbeginn, der ihnen die ihnen zustehende Chance auf eine gesunde Entwicklung nicht verwehrt. Wer die Sprache des Weinens beim Kind versteht, weiß, dass es sich dagegen wehren will, dass ihm Schaden angetan wird. Das Kind bräuchte Anwälte, die sein Recht auf eine geschützte Kindheit vertreten. Paten sollten diese Funktion übernehmen. Und die klassische Familie, die es aber nicht mehr gibt. Unsere Forderungen klingen für viele zu radikal oder viel zu streng. Da müsse es doch realistische Kompromisse geben wie einen Personalschlüssel von 1 zu 6 oder Kinderkrippen ab einem Jahr und Begrenzung des Krippenaufenthalts pro Tag auf acht Stunden. Aber leider sind die Naturgesetze bezüglich der Erfordernisse einer gesunden Entwicklung von Kindern unnachgiebig.

\section{LITERATUR}

Bauer, J. (2015). Selbststeuerung. Die Wiederentdeckung des freien Willens (5. Aufl.). München: Blessing.

Baker, M., Gruber, J. \& Milligan, K. (2017). Long-Run Impacts of a Universal Child Care Program. University of Toronto and NBER

Baker, M. \& Milligan, K. (2016) Boy-Girl Differences in Parental Time Investments: Evidence from Three Countries. Journal of Human Capital, 10(4), 399 - 441.

Baker, M. (2011). Innis Lecture: Universal Early Childhood Interventions: What Is the Evidence Base? Canadian Journal of Economics, 44(4), 1069 - 105.

Baker, M. \& Milligan, K. (2010). Evidence from maternity leave expansions of the impact of maternal care on early child development. Journal of Human Resources, 45(1), 1 - 32.

Baker, M., Gruber, J. \& Milligan, K. (2008). Universal Child Care, Maternal Labor Supply, and Family Well-Being. Journal of Political Economy, 116(4), 709 - 45.

Baker, M., Gruber, J. \& Milligan, K. (2005). Universal Childcare, Maternal Labor Supply, and Family Well-Being. Working Paper no. 11832 (December), NBER, Cambridge, MA.

Belsky, J., \& Pluess, M. (2011). Beyond Adversity, Vulnerability and Resilience: Individual Differences in Developmental Plasticity. In D. Cicchetti \& G. I. Roisman (Eds.), Minnesota Symposium on Child Psychology, Vol.36: The Origins and Organization of Adaptation and Maladaptation (pp. 379-422).

Bischof-Köhler, D. (2010). Kognition, Motivation und Emotion in der frühen Kindheit und im Vorschulalter. In S. Sulz \& S. Höfling (Hrsg.) ... und er entwickelt sich doch! Entwicklung durch Psychotherapie (S. 3-44). München: CIP-Medien.

Böhm, R. (2011). Auswirkungen Frühkindlicher Gruppenbetreuung auf Entwicklung und Gesundheit. Kinderärztliche Praxis, 82, 316 - 21.

Böhm, R. (2013a). Neurobiologische Aspekte der Kleinkindbetreuung. In F. Dammasch \& M. Teising (Hrsg.), Das modernisierte Kind (S. 115 - 128). Frankfurt/M.: Brandes \& Apsel. Böhm, R. (2013b). Stress - das unterschätzte Problem frühkindlicher Betreuung. In C. Haderthauer \& H. Zehetmair (Hrsg.), Was brauchen Kleinkinder, damit Bildung gelingt? Argumente und Materialien zum Zeitgeschehen 83 (S. 27 - 32). München: Hanns-Seidel-Stiftung. 
Böhm, R. (2013c). Das Kleinstkind gehört zu seinen Eltern - die Sicht des Sozialmediziners. In K. Hurrelmann \& T. Schultz (Hrsg.), Staatshilfe für Eltern - Brauchen wir das Betreuungsgeld? (S. 96 - 107). Weinheim/Basel: Beltz-Juventa.

Bowlby, J. \& Holmes, J. (2014). Bindung als sichere Basis: Grundlagen und Anwendung der Bindungstheorie. München: Ernst-Reinhardt.

Brisch, K.-H. (2016). Säuglings- und Kleinkindalter. Stuttgart: Klett-Cotta.

Brisch, K.H. \& Hellbrügge, Th. (Hrsg.) Bindung und Trauma. 2. Aufl. Stuttgart: Klett-Cotta (2006).

Butzmann, E. (2011). Elternkompetenzen stärken. Bausteine für Elternkurse. München: Reinhardt-Verlag.

Bruschweiler-Stern, N. \& Stern, D. (2014). Geburt einer Mutter. Die Erfahrung, die das Leben einer Frau für immer verändert. München: Piper.

Busse, A. \& Gathmann, C. (2018). Free Daycare and its Effects on Children and their Families. Berlin: DIW German Socio-Economic Panel (SOEP). ISSN: 1864-6689 (online)

Eckstein, T., Kappler, G., Datler, W. \& Ahnert, L. (2010). Stressregulation bei Kleinkindern nach Krippeneintritt: Die Wiener Kinderkrippenstudie. Vortrag bei der Jahrestagung der Deutschen Gesellschaft für Psychologie, Bremen.

Fonagy, P., Gergely, G., Jurist, E. L. \& Target, M. (2008). Affektregulierung, Mentalisierung und die Entwicklung des Selbst (3. Aufl.). Suttgart: Klett-Cotta.

Grossmann, K. \& Grossmann, K. E. (2012). Bindungen: Das Gefüge psychischer Sicherheit. Stuttgart: Klett-Cotta.

Gunnar, M. (2001). Effects of early deprivation: Findings from orphanage-reared children. In C. A. Nelson \& M. Luciana (ed.), Handbook of Cognitive Neuroscience (pp. 617-629). Cambridge: MIT Pres.

Heim, C. \& Nemeroff, C.B. (2001). The Role of Childhood Trauma in the Neurobiology of Mood and Anxiety Disorders Preclinical and Clinical Studies. Biological Psychiatry, 49, 1023 - 1039.

Kegan, R. (1986). Die Entwicklungsstufen des Selbst. München: Kindt.

Maaz, H.-J. (2017). Das falsche Leben (3. Aufl.). München: C. H. Beck.

Mischel, W. (2015). Der Marshmallow-Test. München: Siedler-Verlag.

Nemeroff, C.B., Widerlov, E. \& Bissette, G. (1984). Elevated concentrations of CSF corticotropin-releasing factor-like immunoreactivity in depressed patients. Science, 226(4680), 1342-1344.

Nemeroff, C. B. (1996). The corticotropin releasing factor (CRF) hypothesis of depression: new findings an new directions. Mol Psychiatry, 1, 336-342.

Rass, E. (2017). Bindung und Sicherheit im Lebenslauf: Psychodynamische Entwicklungspsychologie. Stuttgart: Klett-Cotta.

Rauschenbach, T., Schilling, M. \& Meiner-Teubner, C. (2017). Plätze. Personal. Finanzen - der Kita-Ausbau geht weiter Zukunftsszenarien zur Kindertages- und Grundschulbetreuung in Deutschland Version 2-2017. Dortmund: Eigenverlag Forschungsverbund DJI/TU.

Roisman, G.I. (2009). Adult attachment: Toward a rapprochement of methodological cultures. Current Directions in Psychological Science, 18(2), 122 - 126.

Scheerer, A. K. (2014). Bindung und Aggression bei den Mosuo - einer matrilinearen Gesellschaft in China. Kinderanalyse, 23 (1), 41-62. 
Schmitt, J., Rossa, K., Rüdiger, M., Reichert, J., Schirutschke, M. et al. (2015). Determinanten der psychischen Gesundheit im Einschulungsalter - Ergebnisse einer populationsbezogenen Untersuchung in Dresden. Kinder- und Jugendarzt, 46(6), 312-325.

Schore, A. N. (2012). Bindung und die rechtshemisphärische Regulation. In E. Rass (Hrsg.), Alan Schore: Schaltstellen der Entwicklung (S. 87-110). Stuttgart: Klett-Cotta.

Sigman, A. (2011): Mother superior? The Biological Effects of Day Care. The Biologist, 58 (3), 28-32.

Stadler, R. (2014). Vater, Mutter, Staat: Das Märchen vom Segen der Ganztagsbetreuung. Wie Politik und Wirtschaft die Familie zerstören. München: Ludwig.

Stüber, N. \& Roth, G. (2017). Die erste Bindung. Wie Eltern das Gehirn ihrer Kinder prägen. Stuttgart: Klett-Cotta.

Sulz, S. (2012). Als Sisyphus seinen Stein losließ oder: Verlieben ist verrückt! Ein psychologisches Lesebuch über menschliche Überlebensformen und individuelle Entwicklungschancen (6. Aufl.). München: CIP-Medien.

Sulz, S. K. D. (2017a). Gute Verhaltenstherapie lernen und beherrschen - Band 1: Verhaltenstherapie-Wissen: So gelangen Sie zu einem tiefen Verständnis des Menschen und seiner Symptome. München: CIP-Medien.

Sulz, S. K. D. (2017b). Gute Verhaltenstherapie lernen und beherrschen - Band 2: Verhaltenstherapie-Praxis: Alles was Sie für eine gute Therapie brauchen. München: CIP-Medien.

Sulz, S. K. D. \& Höfling, S. (Hrsg.). (2010).... und er entwickelt sich doch! Entwicklung durch Psychotherapie. München: CIP-Medien.

Textor, M.R. (2014). Bildungs- und Erziehungspartnerschaft in Kindertageseinrichtungen. Hamburg: BoD.

Tietze, W., Becker-Stoll, F., Bensel, J., Eckhardt, A. G., Haug-Schnabel, G., Kalicki, B. \& Keller, H. (2013). Nationale Untersuchung zur Bildung, Betreuung und Erziehung in der frühen Kindheit (NUBBEK). Verlag am Netz.

Utari-Witt, H. (2018). Bindung, Affektregulation, Strukturbildung und die Bedeutsamkeit der frühen Elternschaft. Vortrag auf dem dgkjf-Symposium „Kinder brauchen keine Kita" am 23.2.2018 in München

Vandell, D. L., Belsky, J., Burchinal, M., Steinberg, L. \& Vandergrift, N./NICHD Early Child Care Research

Network (2010). Do effects of early child care extend to age 15 years? Results From the $\mathrm{NICHD}$

Study of Early Child Care and Youth Development. Child Development, 81 (3), 737-756.

Vermeer, H. \& van Ijzendoorn, J. (2006). Children's Elevated Cortisol Levels at Daycare: A Review and Meta-Analysis. Early Childhood Research Quarterly, 21, 390 - 401.

Wulfes, N. \& Schulz, W. (2017). Der Einfluss außerfamiliärer frühkindlicher Betreuung auf die Entwicklung von Verhaltensauffälligkeiten im Jugendalter. Vortrag am 26.5.2017 35. Symposium der Fachgruppe Klinische Psychologie und Psychotherapie der Deutschen Gesellschaft für Psychologie.

Zepf, S. \& Seel, D. (2017). Würden sich kleine Kinder für ihre Betreuung Kitas ausdenken? Kinderanalyse, 25 (3), xx-xxx. Verfügbar unter: https://www.kinderanalyse.de/article/ ka_2017_03_0203-0230_0203_01 
